\title{
Bootstrap Methods for Bias Correction and Confidence Interval Estimation for Nonlinear Quantile Regression of Longitudinal Data
}

Andreas Karlsson

Division of Statistics
Research Report 2006:2

Research Report Series ISSN 1403-7572

Department of Information Science P.O. Box 513 SE-751 20 Uppsala, Sweden 


\title{
Bootstrap Methods for Bias Correction and Confidence Interval Estimation for Nonlinear Quantile Regression of Longitudinal Data
}

\author{
Andreas Karlsson* \\ Division of Statistics, Department of Information Science, Uppsala University
}

April 6, 2006

\begin{abstract}
This paper examines the use of bootstrapping for bias correction and confidence interval calculations for a weighted nonlinear quantile regression estimator, adjusted to the case of longitudinal data. Different weights, bootstrap methods and types of confidence intervals are used and compared by computer simulation using a four-parameter logistic growth function and error terms following an AR(1) model. Finally the methods are applied to a data set with growth patterns of two genotypes of soybean. It is found that the bias correction reduces the bias, but has the disadvantage of increasing the risk of getting crossing quantile regression curves, and that the bootstrap percentile method and the normal approximation method perform well when used without bias correction. When increasing the autocorrelation in the $\mathrm{AR}(1)$ model, the length of the confidence intervals decreases.
\end{abstract}

Key words: bias reduction, dependent errors, median regression, panel data, repeated measures, simulation study

2000 Mathematics Subject Classification: Primary 62F40, 62J02; Secondary 62M10, 62P10

\section{Introduction}

Longitudinal or repeated measurement data is defined by the characteristic that there are several subjects participating in the study and each subject is measured several times at several time points. This distinguishes it from cross-sectional data, where each subject is observed only once and at one specified time point. The characteristic of having several time series, which often are quite short, also distinguishes longitudinal data from ordinary time series, where usually have only one single long series is studied. Longitudinal data often occur in such applied sciences as medicine and agricultural science. The focus of interest for the scientist is often in how some variable changes over time, for example the pattern of growth for the subjects.

\subsection{Analysis of longitudinal data using quantile regression}

The analysis of longitudinal data has usually been based on using the mean as the location measure (see Vonesh and Chinchilli, 1997, Davis, 2002 or Diggle et al., 2002, for details about different methods for analyzing longitudinal data). However, the mean has the drawback of being a bad measure of centrality

\footnotetext{
* Current Address: Division of Statistics, Department of Information Science, Kyrkogårdsgatan 10, P.O. Box 513, S - 75120 Uppsala, Sweden. Email Address: andreas.karlsson@dis.uu.se. Thanks to Dr. Johan Lyhagen, Professor Rolf Larsson, Dr. Jonas Andersson and Dr. Dag Sörbom for their valuable comments and suggestions.
} 
for heavily skewed data. An alternative is to use the median as the measure of centrality. For longitudinal data regression analysis is a natural choice to use, with time as an independent variable and the variable of interest as the dependent variable, and thus mean or median regression could be used. Median regression is in turn a special case of quantile regression, introduced in the seminal paper by Koenker and Bassett (1978), which can be used to calculate regression curves for any quantile. For overviews of quantile regression, see the monograph by Koenker (2005) as well as the articles by Buchinsky (1998) and Yu et al. (2003).

By calculating regression curves for several different quantiles one gets a distribution of quantile regression curves which together show the distribution of the longitudinal data for each time point, conditional on the specific time points, and the possible change of the distribution over time. Thus quantile regression curves can be used to study the development over time of the distribution of the longitudinal data, and not only the development of the mean or median over time. This gives the analyzer a much more complete picture of the data set. By comparing the values of an individual subject with the quantile regression curves it is easily seen how the individual subject performs compared to the overall performance of the sample. This can be very useful in some instances. In medical applications, for example, a subject with values above or below a certain quantile regression curve may be identified as possibly suffering from some pathological condition.

\subsection{Motivation for using bootstrap with quantile regression for longitudinal data}

A fundamental goal of statistics is to make inferences about parameters by for example calculating confidence intervals and conducting hypothesis tests. A drawback of using median regression instead of mean regression is that the statistical properties are less tractable, which makes it harder to make statistical inference about the parameters. This characteristic is of course also transferred from the median regression case to the general quantile regression case. For longitudinal data the observations from different subjects are usually assumed to be independent, while the different observations from the same subject are correlated. This special structure of the longitudinal data with partly dependent observations makes it harder to make statistical inferences. Making inferences for the quantile regression case applied to longitudinal data is thus even harder. Also, the fact that one in applied sciences neither knows the distribution of the underlying population nor the correlation structure of it implies that one needs to use robust methods for making inferences about the quantile regression parameters.

The bootstrap (Efron, 1979) is a powerful robust method that can be applied to complicated data sets where the statistical properties of the applied estimator are hard to derive. Also, as shown by Karlsson (2005), the estimates from a quantile regression estimation of longitudinal data are somewhat biased, and a method for reducing this bias would be useful. A popular method to reduce the bias of a biased estimator is to use a bootstrap method to estimate the bias of an estimate and then use this to correct the biased estimate. The bootstrap is thus a natural candidate to use both for making inferences and reduce the bias in the case of nonlinear quantile regression for longitudinal data.

\subsection{Earlier work on quantile regression for longitudinal data}

The research about using quantile regression estimation in the analysis of longitudinal data is only in its infancy, with only a few articles published. Jung (1996) proposed a quasi-likelihood method for median regression estimation in the case of longitudinal data, derived its asymptotic properties and showed that median regression with the working assumption of independence gives consistent estimates of the median regression parameters in the case of longitudinal data. Lipsitz et al. (1997) considered the more general 
quantile regression method applied to longitudinal data with missing observations and showed that if it is properly weighted it gives consistent parameter estimates even in this case, if the missing observations are missing at random (MAR) and the model for the missing data is correctly specified. He and Kim (2002) examined semiparametric models for longitudinal data and concluded that it is preferable to use the working assumption of independence when estimating a linear median regression in this case. He et al. (2003) compared three linear median regression estimators for longitudinal data and concluded that the estimator of Koenker and Bassett (1978) using the working assumption of independence is to be preferred. Koenker (2004) studied the asymptotic properties of a penalized weighted linear quantile regression function for longitudinal data. Yu (2004) considered a nonparametric method for quantile regression analysis of longitudinal data and derived its asymptotic properties. Finally Karlsson (2005) suggested a weighted nonlinear quantile regression estimator of the type of Koenker and Bassett (1978) for longitudinal data and examined its small sample properties for different weights through simulation methods.

\subsection{Earlier work on using bootstrap with quantile regression}

Buchinsky (1995) performed a simulation study where he examined the performance of several estimators of the asymptotic covariance matrix for linear quantile regression and censored quantile regression models for cross section data, and from these covariance matrices he constructed confidence intervals. Among the estimators examined were the design matrix bootstrap, the error bootstrap and the sigma bootstrap, as well as three non-bootstrap based estimators. His conclusion was that the design matrix bootstrap was the best estimator overall and was to be preferred. This bootstrap estimator was also applied to an examination of changes in the U.S. wage structure in Buchinsky (1994).

Hahn (1995), again for the cross section case, examined the asymptotic properties of the residual based bootstrap method for linear quantile regression, for both deterministic and random regressors. He showed that the bootstrap distribution converges weakly to the limiting distribution of the quantile regression estimator in probability and concluded that the bootstrap percentile method gives confidence intervals that have asymptotically correct coverage probabilities. Bootstrap methods for quantile regression have also been examined by Fitzenberger (1997), who looked at the use of moving blocks bootstrap for linear quantile regression, and by Horowitz (1998), who examined bootstrap methods for a smoothed median regression estimator.

\subsection{Purpose of this paper}

For the case of longitudinal data bootstrapping has been used by Lipsitz et al. (1997) as well as by He et al. (2003) for estimating the standard errors of the parameter estimates in quantile and median regression models for longitudinal data. However, the performance and usefulness of bootstrapping for quantile regression of longitudinal data has, to our knowledge, never been examined. There is thus a need for such an evaluation, which will be done in this paper. The quantile regression estimation method used is a weighted version of the method of Koenker and Bassett (1978), adjusted to the case of longitudinal data. In this paper the performance of this weighted estimator will be examined by simulation methods, comparing several bootstrap variants for the case of nonlinear longitudinal data. The problems to be examined are a) reducing the bias of the quantile regression estimators and b) calculating confidence intervals for the quantile regression parameters. 


\subsection{Outline of this paper}

This paper is organized as follows. In Section 2 the model and methodology for weighted quantile regression estimation of longitudinal data is introduced. Section 3 introduces bootstrapping and its application to the case of weighted quantile regression for longitudinal data, while Section 4 gives the design of the simulation study. In Section 5 and 6 the results and analysis of the simulation study are given for the cases of bias reduction and confidence interval calculations, respectively. Section 7 contains an application of bootstrap based bias reduction and confidence interval calculations to a real data set with growth patterns of two different genotypes of soybean. Finally, in Section 8 the results and the conclusions that can be drawn of the paper are discussed and the article is closed.

\section{Quantile regression model and estimation methods}

To start with some notations need to be introduced (cf. Karlsson, 2005, and Diggle et al., 2002). Let (i,t) denote observation $t=1, \ldots, T_{i}$ of subject $i=1, \ldots, n$, observed at time $\tau_{i t}$. Then $y_{i t}$ denotes a response variable for observation $(i, t)$ and $\mathbf{y}_{i}=\left(y_{i 1}, \ldots, y_{i T_{i}}\right)^{\prime}$ the corresponding column vector containing all $T_{i}$ response variables for subject $i$. There is thus a total of $N=\sum T_{i}$ observations in the data set. Denote by $x_{i t k}$ explanatory variable $k=1, \ldots, K$ for $y_{i t}$, by $\mathbf{x}_{i t}=\left(x_{i t 1}, \ldots, x_{i t K}\right)^{\prime}$ the column vector containing all $K$ explanatory variables for $y_{i t}$ and by $\mathbf{X}_{i}=\left(\mathbf{x}_{i 1}, \ldots, \mathbf{x}_{i T_{i}}\right)^{\prime}$ the corresponding $T_{i} \times K$ matrix containing all explanatory variables for $\mathbf{y}_{i}$. Also, let $\varepsilon_{i t}$ denote the unknown error term for $y_{i t}$, with $\varepsilon_{i}=\left(\varepsilon_{i 1}, \ldots, \varepsilon_{i T_{i}}\right)^{\prime}$ being the corresponding column vector containing all $T_{i}$ unknown error terms for $\mathbf{y}_{i}$.

The general regression model (linear or nonlinear) for longitudinal data can then be written as

$$
y_{i t}=f\left(\mathbf{x}_{i t}, \boldsymbol{\beta}\right)+\varepsilon_{i t}, \quad i=1, \ldots, n, t=1, \ldots, T_{i},
$$

or, in matrix form,

$$
\mathbf{y}_{i}=\mathbf{f}\left(\mathbf{X}_{i}, \boldsymbol{\beta}\right)+\varepsilon_{i}, \quad i=1, \ldots, n,
$$

where $\mathbf{f}\left(\mathbf{X}_{i}, \boldsymbol{\beta}\right)=\left(f\left(\mathbf{x}_{i 1}, \boldsymbol{\beta}\right), \ldots, f\left(\mathbf{x}_{i T_{i}}, \boldsymbol{\beta}\right)\right), f\left(\mathbf{x}_{i t}, \boldsymbol{\beta}\right)$ denotes a known response functions (linear or nonlinear) and $\boldsymbol{\beta}=\left(\beta_{1}, \ldots, \beta_{h}, \ldots, \beta_{H}\right)^{\prime}$ denotes a column vector containing $H$ unknown parameters. As can be seen, this is a form of the marginal analysis model for longitudinal data discussed in Diggle et al. (2002).

Since this is a model for longitudinal data the vectors of error terms $\varepsilon_{1}, \ldots, \varepsilon_{n}$ from the $n$ different subjects are assumed to be independent, while the error terms $\varepsilon_{i 1}, \ldots, \varepsilon_{i T_{i}}$ of vector $\varepsilon_{i}$ from subject $i$ are assumed to be correlated. Also, note that the model does not assume that the subjects in the longitudinal data set have equally many observations, since the values of $T_{i}$ may vary between subjects. However, when there are in fact equally many observations, the number of observations will be denoted by $T$, i.e., in that case is $T=T_{1}=\cdots=T_{n}$.

\subsection{Model specification}

Many linear or nonlinear functions $f\left(\mathbf{x}_{i t}, \boldsymbol{\beta}\right)$ can be used in $(2.1)$, but this paper will be confined to the use of the four-parameter logistic growth function

$$
f\left(\mathbf{x}_{i t}, \boldsymbol{\beta}\right)=\beta_{1}+\frac{\beta_{2}-\beta_{1}}{1+\exp \left(\beta_{4}\left(x_{i t}-\beta_{3}\right)\right)},
$$

where $x_{i t}=\tau_{i t}$, i.e., time is the only explanatory variable used. The regression parameters $\beta_{1}$ and $\beta_{2}$ give the negative and positive asymptotic values of the response variable, $\beta_{3}$ gives the value of $x$ 
at $\left|\beta_{2}-\beta_{1}\right| / 2$, i.e., the $E C_{50}$ value, while the slope parameter $\beta_{4}$ governs the steepness of the growth curve.

\subsection{Weighted quantile regression}

To estimate a weighted quantile regression for longitudinal data, let $w_{i t}$ denote the weight for observation $(i, t)$ and $\mathbf{W}_{i}$ a symmetric $T_{i} \times T_{i}$ matrix of weights for subject $i$, with elements $w_{i t t^{\prime}}, t, t^{\prime}=1, \ldots, T_{i}$, for observation $t$. Also, for $0<q<1$, where $q$ denotes a quantile, let

$$
\rho_{q}\left(y_{i t}-f\left(\mathbf{x}_{i t}, \boldsymbol{\beta}\right)\right)=\left(y_{i t}-f\left(\mathbf{x}_{i t}, \boldsymbol{\beta}\right)\right)\left(q-I\left\{y_{i t}-f\left(\mathbf{x}_{i t}, \boldsymbol{\beta}\right)<0\right\}\right)
$$

where $I\{\bullet\}$ denotes the indicator function taking the value 1 if $\{\bullet\}$ is true and 0 otherwise, with the corresponding column vector of length $T_{i}$ being

$$
\boldsymbol{\rho}_{q}\left(\mathbf{y}_{i}-\mathbf{f}\left(\mathbf{X}_{i}, \boldsymbol{\beta}\right)\right)=\left(\rho_{q}\left(y_{i 1}-f\left(\mathbf{x}_{i 1}, \boldsymbol{\beta}\right)\right), \ldots, \rho_{q}\left(y_{i T_{i}}-f\left(\mathbf{x}_{i T_{i}}, \boldsymbol{\beta}\right)\right)\right)^{\prime} .
$$

The weighted quantile regression estimate $\widehat{\boldsymbol{\beta}}(q)=\left(\widehat{\beta}_{1}(q), \ldots, \widehat{\beta}_{h}(q), \ldots, \widehat{\beta}_{H}(q)\right)^{\prime}$ of $\boldsymbol{\beta}(q)$, the $q$ th quantile regression of $\boldsymbol{\beta}$ in (2.1), can then be computed by the formula

$$
\widehat{\boldsymbol{\beta}}(q)=\min _{\boldsymbol{\beta}} \sum_{i=1}^{n} \sum_{t=1}^{T_{i}} w_{i t} \rho_{q}\left(y_{i t}-f\left(\mathbf{x}_{i t}, \boldsymbol{\beta}\right)\right),
$$

or, in matrix form,

$$
\begin{aligned}
\widehat{\boldsymbol{\beta}}(q) & =\min _{\boldsymbol{\beta}} \sum_{i=1}^{n} \mathbf{1}_{T_{i}}^{\prime} \mathbf{W}_{i} \boldsymbol{\rho}_{q}\left(\mathbf{y}_{i}-\mathbf{f}\left(\mathbf{X}_{i}, \boldsymbol{\beta}\right)\right) \\
& =\min _{\boldsymbol{\beta}} \sum_{i=1}^{n} \sum_{t=1}^{T_{i}} \sum_{t^{\prime}=1}^{T_{i}} w_{i t t^{\prime}} \rho_{q}\left(y_{i t}-f\left(\mathbf{x}_{i t}, \boldsymbol{\beta}\right)\right),
\end{aligned}
$$

where $\mathbf{1}_{T_{i}}=(1, \ldots, 1)^{\prime}$ is a column vector of 1's with length $T_{i}$ (see Karlsson, 2005).

Note that when all $\mathbf{W}_{i}$ matrices are diagonal with weights $w_{i t}, t=1, \ldots, T_{i}$, as diagonal elements Estimator (2.7) is the same as Estimator (2.6) and that the special case $q=0.5$ is the median regression estimator. As can be seen, Estimators (2.6) and (2.7) are weighted generalizations to the case of longitudinal data of the quantile regression estimator introduced in Koenker and Bassett (1978).

\subsubsection{Weights}

There are of course many weights that could be used for Estimators (2.6) and (2.7). Karlsson (2005) examined the use of different weights $w_{i t}$ and $\mathbf{W}_{i}$ based on residuals $e_{i t}$ computed by first estimating $\widehat{\boldsymbol{\beta}}(0.5)$ when $w_{i t}=1$, i.e.,

$$
\widehat{\boldsymbol{\beta}}(0.5)=\min _{\boldsymbol{\beta}} \sum_{i=1}^{n} \sum_{t=1}^{T_{i}} \rho_{0.5}\left(y_{i t}-f\left(\mathbf{x}_{i t}, \boldsymbol{\beta}\right)\right),
$$

and then with the help of this $\widehat{\boldsymbol{\beta}}(0.5)$ calculated the residuals as

$$
e_{i t}=y_{i t}-f\left(\mathbf{x}_{i t}, \widehat{\boldsymbol{\beta}}(0.5)\right), \quad i=1, \ldots, n, t=1, \ldots, T_{i} .
$$

To define the weights, let the $T \times T$ estimated covariance matrix $\mathbf{S}$ with elements $s_{t t^{\prime}}, t, t^{\prime}=1, \ldots, T$, be defined as 


$$
\mathbf{S}=\frac{1}{n}\left(\mathbf{E}^{\prime} \mathbf{E}-\frac{1}{n} \mathbf{E}^{\prime} \mathbf{1}_{n} \mathbf{1}_{n}^{\prime} \mathbf{E}\right),
$$

where $\mathbf{E}=\left(\mathbf{e}_{1}, \ldots, \mathbf{e}_{n}\right)$ is a $n \times T$ matrix of residuals $\mathbf{e}_{i}=\left(e_{i 1}, \ldots, e_{i T_{i}}\right)$ and $\mathbf{1}_{n}=(1, \ldots, 1)^{\prime}$ is a column vector of 1 's with length $n$. Also, define $s_{i}^{2}$ as

$$
s_{i}^{2}=\frac{1}{T_{i}} \sum_{t=1}^{T_{i}}\left(e_{i t}-\bar{e}_{i}\right)^{2}, \quad i=1, \ldots, n,
$$

where

$$
\bar{e}_{i}=\frac{1}{T_{i}} \sum_{t=1}^{T_{i}} e_{i t}, \quad i=1, \ldots, n .
$$

Then, based on the results of Karlsson (2005), the following three weights will be used:

i. $w_{i t}=1$,

ii. $w_{i t}=\frac{1}{\sqrt{s_{i}^{2}}}$,

iii. $\mathbf{W}_{i}=\left(\frac{1}{\left|s_{t t^{\prime}}\right|}\right)$, i.e., $w_{i t t^{\prime}}=1 /\left|s_{t t^{\prime}}\right|$.

\section{Bootstrap based bias correction and confidence interval esti- mation}

Let $\widehat{\beta}_{h b}^{*}(q)$ denote bootstrap estimate $b=1, \ldots, B$ of $\beta_{h}(q)$, the $q$ th quantile regression of $\beta_{h}$, with $\widehat{\boldsymbol{\beta}}_{b}^{*}(q)$ denoting the corresponding $H \times 1$ vector of bootstrap estimates. Then the $B$ bootstrap estimates together form an estimate of the sampling distribution of $\widehat{\beta}_{h}(q)$. By estimating the bias of this sampling distribution one gets bootstrap estimates of the bias of the estimated quantile regression parameter $\widehat{\beta}_{h}(q)$. Similarly, confidence intervals for the quantile regression parameter $\beta_{h}(q)$ can be computed from this bootstrap distribution.

\subsection{Bias correction}

The straightforward method to compute a bootstrap estimated bias is to use

$$
\widehat{\operatorname{bias}}\left(\widehat{\beta}_{h}(q)\right)=\frac{1}{B} \sum_{b=1}^{B} \widehat{\beta}_{h b}^{*}(q)-\widehat{\beta}_{h}(q),
$$

and the bias corrected parameter estimate $\widehat{\beta}_{h}^{c}(q)$ is then given by

$$
\widehat{\beta}_{h}^{c}(q)=\widehat{\beta}_{h}(q)-\widehat{\operatorname{bias}}\left(\widehat{\beta}_{h}(q)\right)
$$

\subsection{Bootstrap based confidence intervals}

Bootstrap based confidence intervals for the quantile regression parameter $\beta_{h}(q)$ can be computed in several different ways. In this article two different approaches will be used. The first is based on a normal approximation, which can be justified by the results of Oberhofer and Haupt (2005), who showed that the unweighted version of the nonlinear quantile regression estimator (2.7) is asymptotically normally distributed for a nonlinear quantile regression model with heteroscedastic and weakly dependent errors. 
An alternative to the normal approximation method is to use some version of the percentile method introduced by Efron (1979). For an overview of the calculations of bootstrap based confidence intervals, see Carpenter and Bithell (2000), and for a detailed account, see e.g. Chapter 5 of Davison and Hinkley (1997).

\subsubsection{Normal approximation method}

For the normal approximation method a $100(1-2 \alpha)$ percent confidence interval for $\beta_{h}(q)$ is calculated as

$$
\widehat{\beta}_{h}(q) \pm Z_{\alpha} \sqrt{\widehat{\operatorname{Var}}^{*}\left(\widehat{\beta}_{h}(q)\right)}
$$

where $\widehat{\operatorname{Var}}^{*}\left(\widehat{\beta}_{h}(q)\right)$ is the bootstrap estimated variance computed as

$$
\widehat{\operatorname{Var}}^{*}\left(\widehat{\beta}_{h}(q)\right)=\frac{1}{B-1} \sum_{b=1}^{B}\left(\widehat{\beta}_{h b}^{*}(q)-\frac{1}{B} \sum_{b=1}^{B} \widehat{\beta}_{h b}^{*}(q)\right)^{2} .
$$

Confidence intervals based on this normal approximation method will be denoted $C I_{N}$.

A variant of the normal approximation method is the bias corrected normal approximation method,

$$
\widehat{\beta}_{h}^{c}(q) \pm Z_{\alpha} \sqrt{\widehat{\operatorname{Var}}^{*}\left(\widehat{\beta}_{h}(q)\right)}
$$

where $\widehat{\beta}_{h}^{c}(q)$ is given by (3.2). Confidence intervals based on this method will be denoted $C I_{N B C}$.

\subsubsection{Bias corrected percentile method}

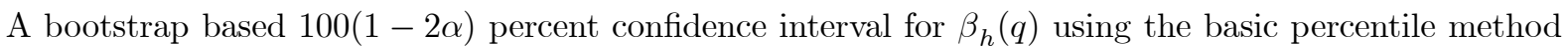
is given by

$$
\widehat{\beta}_{h(\alpha(B+1))}^{*}(q) \leq \beta_{h}(q) \leq \widehat{\beta}_{h((1-\alpha)(B+1))}^{*}(q),
$$

where $\widehat{\beta}_{h(b)}^{*}(q)$ is the $b$ th order statistic of the $B$ bootstrap replicates $\widehat{\beta}_{h(1)}^{*}(q) \leq \cdots \leq \widehat{\beta}_{h(B)}^{*}(q)$. Confidence intervals based on this percentile method will be denoted $C I_{P}$.

Now, let $P^{*}\left(\widehat{\beta}_{h}^{*}(q) \leq \widehat{\beta}_{h}(q)\right)$ denote the proportion of $\widehat{\beta}_{h}^{*}(q)$ 's in the bootstrap sample that have a value lower than the value of the parameter estimate $\widehat{\beta}_{h}(q)$, and let $z_{0}$ be defined as

$$
z_{0}=\Phi^{-1}\left(P^{*}\left(\widehat{\beta}_{h}^{*}(q) \leq \widehat{\beta}_{h}(q)\right)\right),
$$

where $\Phi$ denotes the cumulative distribution function (CDF) of the standard normal distribution. Also, let $\widetilde{\alpha}_{1}$ and $\widetilde{\alpha}_{2}$ be defined as

$$
\widetilde{\alpha}_{1}=\Phi\left(2 z_{0}+z_{\alpha}\right)
$$

and

$$
\widetilde{\alpha}_{2}=\Phi\left(2 z_{0}+z_{1-\alpha}\right)
$$

respectively.

A $100(1-2 \alpha)$ percent confidence interval for $\beta_{h}(q)$ is then given by 


$$
\widehat{\beta}_{h\left(\widetilde{\alpha}_{1}(B+1)\right)}^{*}(q) \leq \beta_{h}(q) \leq \widehat{\beta}_{h\left(\widetilde{\alpha}_{2}(B+1)\right)}^{*}(q) .
$$

Confidence intervals based on this bias corrected percentile method will be denoted $C I_{P B C}$. For details about this method, see Section 4.1.3 in Shao and Tu (1995) or Section 3.4 in Manly (1997).

\section{Bootstrapping quantile regressions for longitudinal data}

When bootstrapping data from a weighted quantile regression of longitudinal data one has to take care of the special characteristics for this kind of data, i.e., the weighting method used and the dependence structure of the data. The simplest bootstrap method is to disregard the dependence between the observations for a subject and proceed as if all observations and subjects where independent. However, a major drawback is that it can only be used with weight method i, $w_{i t}=1$, which just like this bootstrap method ignores the fact that the observations are dependent. Weight method ii can not be used since $s_{i}^{2}$ calculated from the bootstrap sample could be zero for this case, which would mean that $w_{i t}=1 / \sqrt{s_{i}^{2}}$ would involve division by zero. This is true also for $s_{t t^{\prime}}$ in weight method iii, $\mathbf{W}_{i}=\left(1 /\left|s_{t t^{\prime}}\right|\right)$, and for this weight there is also the complication that it is required that each bootstrap sample should include equally many observations $t$ for each subject $i$, which not is possible to bring about.

Since longitudinal data are correlated it would possibly be a good idea to use bootstrap methods that preserves and makes use of the correlation structure of the data. Such a method is the block bootstrap method, where blocks of consecutive observations are resampled, instead of resampling single observations. By using this method the dependence structure in each block is retained. See Lahiri (2003) for details about different block bootstrap methods. For longitudinal data, where it is assumed that the subjects $i$ are independent but the consecutive observations $t$ for an individual subject are dependent, resampling of blocks consisting of all observations for a particular individual is the natural block bootstrap method to use.

\subsection{Paired bootstrap with independence resampling}

This method disregards the correlation between the observations for a subject and proceeds as if all observations and subjects where independent. To use this method $N=\sum T_{i}$ pairs $\left(y_{i t}, \mathbf{x}_{i t}^{\prime}\right)$ of observations with response and independent variables are resampled (with replacements) to form the paired bootstrap sample $\left(y_{1}^{*}, \mathbf{x}_{1}^{\prime *}\right), \ldots,\left(y_{N}^{*}, \mathbf{x}_{N}^{\prime *}\right)$. These are then used to compute the bootstrap estimate $\widehat{\boldsymbol{\beta}}_{b}^{*}(q)$ of $\boldsymbol{\beta}(q)$ with the formula

$$
\widehat{\boldsymbol{\beta}}_{b}^{*}(q)=\min _{\boldsymbol{\beta}} \sum_{j=1}^{N} w_{j}^{*} \rho_{q}\left(y_{j}^{*}-f\left(\mathbf{x}_{j}^{*}, \boldsymbol{\beta}\right)\right), \quad b=1, \ldots, B,
$$

where $w_{j}^{*}$ is the bootstrap quantile regression weight. By repeating this process $B$ times are the $B$ bootstrap estimates $\widehat{\boldsymbol{\beta}}_{b}^{*}(q)$ of $\boldsymbol{\beta}(q)$ received. This method will be called paired bootstrap with independence resampling, $P B_{I}$, in this paper.

An advantage of this bootstrap method is that it is simple to apply. However, as noted above, a major drawback is that it can only be used with weight method i, $w_{i t}=1$. Thus only $w_{j}^{*}=1$ can be used in Formula (4.1).

\subsection{Paired bootstrap with dependence resampling}

For a block bootstrap method where the blocks consist of all observations for a particular subject $n$ pairs $\left(\mathbf{y}_{i}, \mathbf{X}_{i}\right)$ of observations with response and independent variables, consisting of all observations 
from a subject, are resampled (with replacements). These will form the paired bootstrap sample $\left(\mathbf{y}_{1}^{*}, \mathbf{X}_{1}^{*}\right), \ldots,\left(\mathbf{y}_{n}^{*}, \mathbf{X}_{n}^{*}\right)$, which then can be used to compute the bootstrap estimate $\widehat{\boldsymbol{\beta}}_{b}^{*}(q)$ of $\boldsymbol{\beta}(q)$ with the help of the formula

$$
\widehat{\boldsymbol{\beta}}_{b}^{*}(q)=\min _{\boldsymbol{\beta}} \sum_{j=1}^{n} \mathbf{1}_{T_{j}}^{\prime} \mathbf{W}_{j}^{*} \boldsymbol{\rho}_{q}\left(\mathbf{y}_{i}^{*}-\mathbf{f}\left(\mathbf{X}_{i}^{*}, \boldsymbol{\beta}\right)\right), \quad b=1, \ldots, B,
$$

where $\mathbf{W}_{j}^{*}$ is the bootstrap quantile regression weight matrix. Then, by repeating this process $B$ times are the $B$ bootstrap estimates $\widehat{\boldsymbol{\beta}}_{b}^{*}(q)$ of $\boldsymbol{\beta}(q)$ received. This method will be called paired bootstrap with dependence resampling, $P B_{D}$, in this paper.

For the bootstrap quantile regression weight matrix $\mathbf{W}_{j}^{*}$ in (4.2) all three weight methods i-iii can be used. For weights i and ii $\mathbf{W}_{j}^{*}$ is a diagonal $T_{i} \times T_{i}$ weight matrix with diagonal elements $w_{j t t}^{*}=1$ and $w_{j t t}^{*}=1 / \sqrt{s_{j}^{2 *}}$, respectively, while for weight iii $\mathbf{W}_{j}^{*}=\left(1 /\left|s_{t t^{\prime}}^{*}\right|\right)$, i.e., $w_{j t t^{\prime}}^{*}=1 /\left|s_{t t^{\prime}}^{*}\right|$, where $s_{j}^{2 *}$ and $s_{t t^{\prime}}^{*}$ are computed in an obvious way using the corresponding bootstrap residuals $\mathbf{e}_{j}^{*}$.

\section{$5 \quad$ Simulation study design and results}

A simulation study is conducted to examine the usefulness of bootstrapping for a) reducing the bias of the quantile regression estimates and b) calculating confidence intervals for the quantile regression parameters. This section will introduce the design and data generating process used in the simulation study, while the following two sections present the result of the simulations.

To start with a correlation structure has to be specified for the errors $\varepsilon_{i 1}, \ldots, \varepsilon_{i T}$ in (2.1). This correlation is generated by the $\mathrm{AR}(1)$-model

$$
\varepsilon_{i t}=\rho \varepsilon_{i(t-1)}+u_{i t}, \quad i=1, \ldots, n, t=1, \ldots, T,
$$

with the resulting $\varepsilon_{i t}$ 's transformed to have median 0 and variance 1 . The $\beta$-values in (2.3) also have to be specified. In this paper the following values will be used: $\beta_{1}=10, \beta_{2}=20, \beta_{3}=5$ and $\beta_{4}=-0.5$, while the values of $x_{i t}$ in (2.3) are specified as $x_{i t}=10 \times \frac{t}{T}$. This is in line with the specifications used in Karlsson (2005). For (5.1) the $u_{i t}$ 's will be distributed as standard normal or standard lognormal.

Since the dependence between the error terms $\varepsilon_{i 1}, \ldots, \varepsilon_{i T}$ is a fundamental characteristic of longitudinal data the influence of the strength of the dependence on the performance of the bias estimators should be an important aspect to examine. To do this the cases of no dependence $(\rho=0)$, medium dependence ( $\rho=0.5)$ and strong dependence $(\rho=0.95)$ in the $\operatorname{AR}(1)$ process $(5.1)$ will be compared. Furthermore it is important to examine the performance of the bootstrap for different quantiles and number of subjects $n$ and observations $T$. Thus three different quantiles $q$ will be compared, namely $q=0.5, q=0.75$ and $q=0.90$, while the number of subjects and observations $(n, T)$ used are $(20,5)$ and $(50,10)$. The number of bootstrap replicates $B$ and simulation study replicates $R$ used is set to $B=1000$ and $R=1000$. The simulation study is performed in MATLAB ${ }^{\circledR}$, using a built-in general minimization algorithm.

\subsection{Results for bias correction}

In this section the results for the bias correction using (3.2) are given. The main focus is on the improvements in bias when using $\widehat{\beta}_{h}^{c}(q)$ instead of $\widehat{\beta}_{h}(q)$, where the biases of $\widehat{\beta}_{h}(q)$ and $\widehat{\beta}_{h}^{c}(q)$ are estimated by

$$
\widehat{\operatorname{bias}}\left(\widehat{\beta}_{h R}(q)\right)=\frac{\frac{1}{R} \sum_{r=1}^{R} \widehat{\beta}_{h r}(q)-\beta_{h}(q)}{\left|\beta_{h}(q)\right|} \times 100, \quad h=1, \ldots, H,
$$


Table 5.1: Absolute values of bias, in percent, after correction for bias, and improvement in bias after correction, in percentage points. Mean values taken over all $\rho, \mathrm{n}, \mathrm{T}$ and distributions.

\begin{tabular}{|c|c|c|c|c|c|c|c|c|c|c|}
\hline \multicolumn{3}{|c|}{ bootstrap } & \multicolumn{4}{|c|}{ bias after correction } & \multicolumn{4}{|c|}{ improvement in bias } \\
\hline$q$ & method & weight & $\beta_{1}$ & $\beta_{2}$ & $\beta_{3}$ & $\beta_{4}$ & $\beta_{1}$ & $\beta_{2}$ & $\beta_{3}$ & $\beta_{4}$ \\
\hline \multirow[t]{4}{*}{0.5} & $P B_{I}$ & i & 2.20 & 0.34 & 1.21 & 0.64 & -0.45 & 0.06 & -0.33 & -0.10 \\
\hline & $P B_{D}$ & $\mathrm{i}$ & 1.01 & 0.11 & 0.75 & 0.59 & 0.74 & 0.29 & 0.13 & -0.04 \\
\hline & & ii & 1.84 & 0.61 & 0.97 & 0.66 & 0.34 & 0.06 & -0.04 & 0.29 \\
\hline & & iii & 0.84 & 0.09 & 0.66 & 0.49 & 0.55 & 0.27 & 0.00 & 0.29 \\
\hline \multirow[t]{4}{*}{0.75} & $P B_{I}$ & i & 2.86 & 0.84 & 1.34 & 1.41 & -0.20 & -0.12 & -0.21 & -0.33 \\
\hline & $P B_{D}$ & $\mathrm{i}$ & 1.81 & 0.48 & 1.08 & 0.82 & 0.85 & 0.24 & 0.05 & 0.26 \\
\hline & & ii & 1.83 & 0.77 & 0.89 & 1.32 & 0.74 & -0.07 & 0.03 & 0.57 \\
\hline & & iii & 1.58 & 0.18 & 1.37 & 0.61 & 0.55 & 0.37 & -0.44 & 0.73 \\
\hline \multirow[t]{4}{*}{0.90} & $P B_{I}$ & $\mathrm{i}$ & 3.89 & 0.99 & 2.54 & 3.36 & 0.03 & 0.35 & -1.31 & 1.11 \\
\hline & $P B_{D}$ & i & 2.87 & 0.37 & 2.39 & 1.88 & 1.03 & 0.96 & -1.18 & 2.59 \\
\hline & & ii & 2.80 & 1.39 & 1.43 & 2.21 & 0.89 & -0.30 & -0.33 & 0.93 \\
\hline & & iii & 1.79 & 0.36 & 1.37 & 1.46 & 1.03 & 0.89 & -0.40 & 2.40 \\
\hline
\end{tabular}

and

$$
\widehat{\operatorname{bias}}\left(\widehat{\beta}_{h R}^{c}(q)\right)=\frac{\frac{1}{R} \sum_{r=1}^{R} \widehat{\beta}_{h r}^{c}(q)-\beta_{h}(q)}{\left|\beta_{h}(q)\right|} \times 100, \quad h=1, \ldots, H
$$

respectively. The improvement in bias is then calculated as

$$
\text { bias improvement }=\left|\widehat{\text { bias }}\left(\widehat{\beta}_{h R}(q)\right)\right|-\left|\widehat{\text { bias }}\left(\widehat{\beta}_{h R}^{c}(q)\right)\right|
$$

A negative value for the bias improvement is thus a deterioration.

\subsubsection{Comparison over the different quantiles}

We will first look at the performance of the bootstrap methods $P B_{I}$ and $P B_{D}$ for the different quantiles, $q=0.5, q=0.75$ and $q=0.9$. Table 5.1 gives the mean values of the absolute values of the estimated biases of the $\widehat{\beta}_{h}^{c}(q)$ 's, together with the improvement in the biases, calculated over the normal and lognormal distributions and all values of $\rho, n$ and T. Table A.1 in the Appendix gives more details, with mean values calculated only over $\rho$, thus giving separate values for the normal and lognormal distributions and the different values of $n$ and $T$.

Using the bootstrap method $P B_{I}$, which only can be used with weight $\mathrm{i}$, for correcting the bias does not seem to be a good idea. For 28 of the 48 cases in Table A.1, more than half of the cases, the average estimated bias for the bias corrected parameter estimates $\widehat{\beta}_{h}^{c}(q)$ is larger than that of the parameter estimates that is not bias corrected, $\widehat{\beta}_{h}(q)$. The bias correction based on $P B_{I}$ thus worsens the bias of the quantile regression estimator. Especially the bias correction for the estimates of the $E C_{50}$ parameter $\beta_{3}$ works bad, as is also obvious from Table 5.1, with all bias improvements for this parameter being negative, thus in fact being deteriorations.

When comparing the performance of the bootstrap methods $P B_{I}$ and $P B_{D}$ for weight i in Tables 5.1 and A.1, it is obvious that $P B_{D}$ is to be preferred. From Table 5.1 it is seen that the estimated biases of the $\widehat{\beta}_{h}^{c}(q)$ 's are always lower, and the bias improvements better, for $P B_{D}$ than for $P B_{I}$. In Table A.1, the bias improvements are better for $P B_{D}$ than for $P B_{I}$ in 44 of the 48 cases. Thus, in general, the bootstrap method $P B_{D}$ is to be preferred over $P B_{I}$ for all quantiles, $q=0.5, q=0.75$ and $q=0.9$.

Looking at the three weights used with bootstrap method $P B_{D}$, weights i, ii, and iii, it is seen from Tables 5.1 and A.1 that overall are they all gaining somewhat from using the bias correction, in that the 
Table 5.2: Absolute values of bias, in percent, after correction for bias, and improvement in bias after correction, in percentage points. Mean values taken over all q, n, T and distributions.

\begin{tabular}{|c|c|c|c|c|c|c|c|c|c|c|}
\hline \multicolumn{3}{|c|}{ bootstrap } & \multicolumn{4}{|c|}{ bias after correction } & \multicolumn{4}{|c|}{ improvement in bias } \\
\hline$\rho$ & method & weight & $\beta_{1}$ & $\beta_{2}$ & $\beta_{3}$ & $\beta_{4}$ & $\beta_{1}$ & $\beta_{2}$ & $\beta_{3}$ & $\beta_{4}$ \\
\hline \multirow[t]{4}{*}{0} & $P B_{I}$ & i & 3.67 & 0.62 & 2.29 & 1.90 & 1.31 & 0.80 & -0.43 & 1.57 \\
\hline & $P B_{D}$ & $\mathrm{i}$ & 3.62 & 0.64 & 2.20 & 1.61 & 1.34 & 0.78 & -0.36 & 1.88 \\
\hline & & ii & 3.68 & 0.75 & 1.83 & 0.96 & 0.96 & -0.11 & -0.20 & 1.03 \\
\hline & & iii & 2.73 & 0.36 & 1.98 & 1.22 & 1.25 & 0.87 & -0.37 & 2.13 \\
\hline \multirow[t]{4}{*}{0.5} & $P B_{I}$ & $\mathrm{i}$ & 1.06 & 0.45 & 1.37 & 2.09 & 1.94 & 0.46 & -0.18 & -0.26 \\
\hline & $P B_{D}$ & $\mathrm{i}$ & 1.55 & 0.22 & 1.75 & 1.08 & 1.45 & 0.69 & -0.56 & 0.75 \\
\hline & & ii & 2.10 & 1.09 & 1.19 & 1.77 & 0.90 & -0.20 & -0.09 & 0.57 \\
\hline & & iii & 1.05 & 0.16 & 1.14 & 0.76 & 0.98 & 0.63 & -0.38 & 1.12 \\
\hline \multirow[t]{4}{*}{0.95} & $P B_{I}$ & $\mathrm{i}$ & 4.22 & 1.10 & 1.43 & 1.41 & -3.88 & -0.98 & -1.24 & -0.64 \\
\hline & $P B_{D}$ & $\mathrm{i}$ & 0.51 & 0.11 & 0.27 & 0.60 & -0.17 & 0.01 & -0.08 & 0.18 \\
\hline & & ii & 0.69 & 0.94 & 0.27 & 1.47 & 0.11 & 0.00 & -0.05 & 0.19 \\
\hline & & iii & 0.43 & 0.11 & 0.28 & 0.59 & -0.10 & 0.03 & -0.08 & 0.17 \\
\hline
\end{tabular}

Table 5.3: Quantile regression parameter estimates for quantiles 0.5, 0.75 and 0.9 when $\mathrm{n}=20, \mathrm{~T}=5, \rho$ $=0.5$, with a standard normal distrubution. The parameter estimates are the mean of the 1,000 Monte Carlo replications for weight iii and are used in Figure 1.

\begin{tabular}{c|rrrr|rrrr|} 
& \multicolumn{4}{|c|}{ before bias correction } & \multicolumn{4}{c|}{ after bias correction } \\
$q$ & $\beta_{1}$ & $\beta_{2}$ & $\beta_{3}$ & \multicolumn{1}{c|}{$\beta_{4}$} & $\beta_{1}$ & $\beta_{2}$ & $\beta_{3}$ & \multicolumn{1}{c|}{$\beta_{4}$} \\
\hline 0.5 & 9.617 & 20.162 & 4.908 & -0.504 & 9.813 & 20.044 & 4.923 & -0.492 \\
true values & 10 & 20 & 5 & -0.5 & 10 & 20 & 5 & -0.5 \\
\hline 0.75 & 10.258 & 20.844 & 4.903 & -0.508 & 10.409 & 20.724 & 4.880 & -0.496 \\
true values & 10.675 & 20.675 & 5 & -0.5 & 10.675 & 20.675 & 5 & -0.5 \\
\hline 0.9 & 10.963 & 21.443 & 4.959 & -0.516 & 11.152 & 21.259 & 4.904 & -0.504 \\
true values & 11.282 & 21.282 & 5 & -0.5 & 11.282 & 21.282 & 5 & -0.5 \\
\hline
\end{tabular}

average estimated biases for the $\widehat{\beta}_{h}^{c}(q)$ 's are overall somewhat lower than those of $\widehat{\beta}_{h}(q)$. Only for the estimates of the $E C_{50}$ parameter $\beta_{3}$ for the quantile $q=0.9$ are all three weights having a deterioration in bias. However, the gains from using the bias correction are usually quite small, with a bias improvement of more than one percentage point for only 24 of the 108 cases (22.2 percent) in Table A.1. It is also seen that, in general, the bias improvements increases with increasing $q$, except for parameter $\beta_{3}$, for which the bias improvements decreases with increasing $q$.

Finally, looking at the average bias corrected parameter estimates $\widehat{\beta}_{h}^{c}(q)$ in Table 5.1 it is seen that weight iii generally has the lowest bias, followed by weight $i$, although the differences between the weights are small. The biases are quite small, especially for the median $q=0.5$. It is also seen from Table 5.1 that increasing the value of $q$ in general also increases the bias, so that, in general, the parameter estimates for $q=0.9$ have larger biases than those for $q=0.75$, while the parameter estimates for $q=0.75$ in turn have larger biases than those for $q=0.5$. This agrees with the findings of Karlsson (2005).

\subsubsection{Comparison over the different sizes of correlation}

In this section we will look at the performance of the bootstrap methods $P B_{I}$ and $P B_{D}$ for the different degrees of dependence among the observations from the same subject, with $\rho=0, \rho=0.5$ or $\rho=0.95$ in the $\mathrm{AR}(1)$ process (5.1). Table 5.2 gives the mean values of the absolute values of the estimated biases of the $\widehat{\beta}_{h}^{c}(q)$ 's, together with the improvement in the biases, calculated over the normal and lognormal distributions and all values of $q, n$ and T. Table A.2 in the Appendix gives more details, with mean values calculated only over $q$, thus giving separate values for the normal and lognormal distributions and the different values of $n$ and $T$. 
First, comparing the performance of $P B_{I}$ and $P B_{D}$ for weight i, it is found that overall $P B_{D}$ performs better than $P B_{I}$. In 34 of 48 cases (71 percent) in Table A.2 the improvements in bias are larger, or the deterioration smaller, for $P B_{D}$ than for $P B_{I}$. Especially for the case of strong dependence, $\rho=0.95$, $P B_{D}$ is superior to $P B_{I}$, with better performance in 14 of 16 cases (88 percent) in Table A.2. For all these 14 cases $P B_{I}$ has a deterioration in bias. Notably is the particularly bad performance of $P B_{I}$ for $\beta_{1}$ when $n=20, T=5$ and $\rho=0.95$, with a deterioration in bias of 6.39 percentage points for the normal distribution and 7.02 percentage points for the lognormal distribution.

When comparing the three weights, weights i, ii, and iii, used with the bootstrap method $P B_{D}$, it is found that overall all weights gain somewhat from using the bias correction. However, note from Table 5.2 that for $\beta_{3}$ the biases for all three weights deteriorate for all cases of $\rho$. This gains are largest for $\rho=0$ and smallest for $\rho=0.95$. However, the gains are usually quite small, with a bias improvement of more than one percentage point for only 28 of the 108 cases (25.9 percent) in Table A.2. In fact, the gains for $\rho=0.95$ are so small that they are practically negligible. These results should come as no surprise, since Karlsson (2005) showed that the biases are smallest for $\rho=0.95$, thus making the potential gains small.

Overall weight iii gain most in bias improvement, followed by weight $\mathrm{i}$. This pattern is reflected also in the average bias corrected parameter estimates $\widehat{\beta}_{h}^{c}(q)$ in Table 5.2, where it is found that weight iii generally has the lowest bias, followed by weight $i$, although the differences between the weights are small. As is seen from Table 5.2 also increasing dependence, i.e., larger values of $\rho$, in general implies a decrease in bias. Thus, in general, the parameter estimates for $\rho=0.95$ have smaller biases than those for $\rho=0.5$, and the parameter estimates for $\rho=0.5$ in turn have smaller biases than those for $\rho=0$. This agrees with the findings of Karlsson (2005).

\subsubsection{An example}

As an example of the bias improvements, Table 5.3 gives the parameter estimates of $\beta_{1}, \beta_{2}, \beta_{3}$ and $\beta_{4}$ for weight iii using $P B_{D}$, before and after the bias correction, for quantiles $q=0.5, q=0.75$ and $q=0.90$, using the case of $n=20, T=5, \rho=0.5$, and the $u_{i t}$ 's in (5.1) being standard normal distributed. The table also gives the true parameter values for $\beta_{1}, \beta_{2}, \beta_{3}$ and $\beta_{4}$. Figure 5.1 plots the quantile regression curves, using parameter estimates from the table, before (dashed) and after (dotted) bias correction, together with the true quantile regression curves (solid).

As can be seen from table 5.3 and Figure 5.1, the bias of the parameters are overall decreasing, and is barely discernible in 5.1 for the positive asymptote $\beta_{2}$. It is quite low also for the negative asymptote $\beta_{1}$. Only for $\beta_{3}$ when $q=0.75$ and $q=0.90$, and for $\beta_{4}$ when $q=0.5$, are the biases deteriorating when using the bias correction, although the biases for these cases still are quite small.

\subsection{Results for confidence interval calculation}

In this section the results for the confidence interval calculations using $C I_{N}, C I_{N B C}, C I_{P}$ and $C I_{P B C}$ will be analyzed. This will be done by comparing the true percentage coverages of 95 percent confidence intervals for these four methods, i.e., the percentage of times when the confidence intervals for $\beta_{h}(q)$ covers the true $\beta_{h}(q)$ when the nominal coverage is 95 percent. Besides this we will also compare the lengths of the confidence intervals. Although the comparisons between the lengths of the confidence intervals are strictly correct only if the true coverages are equal, which usually is not the case, the comparisons still give some insights about the performance of the different methods for confidence interval estimation, especially for the case where one does not try to find out the true coverage and then adjust the nominal significance level according to this, which probably is the most common practice in real applications. 


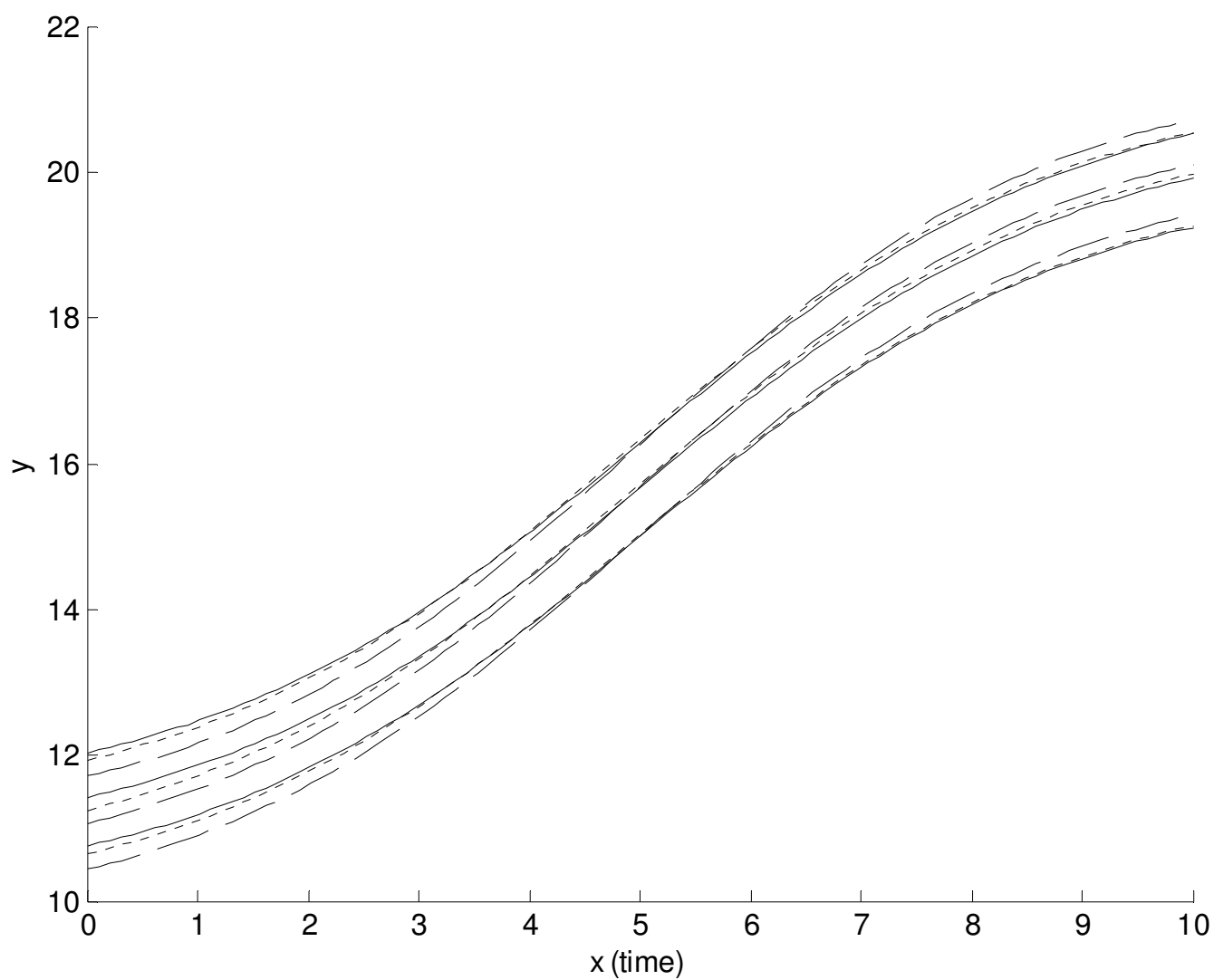

Figure 5.1: Quantile regression curves for quantiles 0.5, 0.75 and 0.90, using parameter estimates for weight iii using $P B_{D}$ before (dashed) and after (dotted) bias correction, together with the true quantile regression curves (solid), for the case of $n=20, T=5, \rho=0.5$ and standard normal distribution.

\subsubsection{Comparison over the different quantiles}

Table 5.4 gives the true coverage of 95 percent confidence intervals using $C I_{N}, C I_{N B C}, C I_{P}$ and $C I_{P B C}$ for the different quantiles, $q=0.5, q=0.75$ and $q=0.9$, calculated as the mean values over the normal and lognormal distributions and all values of $\rho, n$ and T. Tables A.3 - A.6 in the Appendix give more details, with the true coverages calculated as the mean only over $\rho$, thus giving results separately for the normal and lognormal distribution and the different choices of $n$ and $T$.

As is seen from Table 5.4 and Table A.3 in the appendix, all confidence intervals for the normal approximation method $C I_{N}$ using bootstrap method $P B_{I}$ have a true coverage of at least 95 percent at the nominal 95 percent level. This is true also for weight iii using bootstrap method $P B_{D}$, while weight i using $P B_{D}$ has a true coverage of at least 95 percent for all except $\beta_{2}$ when $n=20, T=5$ for quantile $q=0.9$, when the true coverage is 94.99 percent in Table 5.4 and slightly below 95 percent for two cases in Table A.3. Of these three weight i using $P B_{D}$ overall has the true coverage that is closest to the nominal 95 percent. Weight ii, however, is performing bad, with more than half of the confidence intervals in Table 5.4 having true coverages of below 95 percent.

Then, looking at the bias corrected normal approximation method $C I_{N B C}$ in Table 5.4 and Table A.4 in the appendix, it is seen that the confidence intervals based on $P B_{D}$ all perform bad, with all confidence intervals for weights $\mathrm{i}$, ii and iii in Table 5.4 having true coverages below the nominal 95 percent. Bootstrap method $P B_{I}$ with weight i performs better, with only one case in Table 5.4 being below the nominal 95 percent level, but even for this case are 8 of 48 confidence intervals in Table A.4 
Table 5.4: Confidence intervals, true coverage in percent for nominal 95 percent level, normal approximation with and without bias correction together with percentile method with and without bias correction. Mean values taken over all $\rho, \mathrm{n}, \mathrm{T}$ and distributions.

\begin{tabular}{|c|c|c|c|c|c|c|c|c|c|c|}
\hline \multicolumn{3}{|c|}{ bootstrap } & \multicolumn{4}{|c|}{$C I_{N}$} & \multicolumn{4}{|c|}{$C I_{N B C}$} \\
\hline$q$ & method & weight & $\beta_{1}$ & $\beta_{2}$ & $\beta_{3}$ & $\beta_{4}$ & $\beta_{1}$ & $\beta_{2}$ & $\beta_{3}$ & $\beta_{4}$ \\
\hline \multirow[t]{4}{*}{0.5} & $P B_{I}$ & i & 98.34 & 97.70 & 98.23 & 98.03 & 97.09 & 96.29 & 97.20 & 95.71 \\
\hline & $P B_{D}$ & $\mathrm{i}$ & 97.17 & 96.34 & 97.44 & 96.47 & 92.85 & 92.70 & 93.86 & 90.20 \\
\hline & & ii & 94.70 & 89.87 & 94.40 & 92.99 & 88.24 & 84.28 & 88.57 & 83.68 \\
\hline & & iii & 97.84 & 97.48 & 98.11 & 97.48 & 92.73 & 93.12 & 93.63 & 89.18 \\
\hline \multirow[t]{4}{*}{0.75} & $P B_{I}$ & $\mathrm{i}$ & 98.24 & 97.83 & 98.29 & 97.93 & 96.62 & 96.52 & 97.03 & 95.11 \\
\hline & $P B_{D}$ & $\mathrm{i}$ & 97.03 & 96.38 & 97.59 & 96.69 & 92.96 & 93.03 & 94.11 & 90.48 \\
\hline & & ii & 96.43 & 87.02 & 96.04 & 94.51 & 90.56 & 82.16 & 90.51 & 85.44 \\
\hline & & iii & 97.71 & 97.28 & 98.02 & 97.45 & 92.93 & 93.33 & 93.83 & 89.24 \\
\hline \multirow[t]{4}{*}{0.90} & $P B_{I}$ & $\mathrm{i}$ & 97.73 & 96.65 & 98.52 & 98.15 & 95.37 & 95.25 & 96.97 & 94.20 \\
\hline & $P B_{D}$ & $\mathrm{i}$ & 96.96 & 94.99 & 97.73 & 97.13 & 91.94 & 91.72 & 93.62 & 90.10 \\
\hline & & ii & 97.09 & 85.39 & 96.66 & 95.42 & 91.01 & 80.07 & 90.63 & 85.38 \\
\hline & & iii & 97.55 & 96.36 & 98.18 & 97.33 & 92.58 & 92.43 & 93.43 & 88.40 \\
\hline \multicolumn{3}{|c|}{ bootstrap } & \multicolumn{4}{|c|}{$C I_{P}$} & \multicolumn{4}{|c|}{$C I_{P B C}$} \\
\hline$q$ & method & weight & $\beta_{1}$ & $\beta_{2}$ & $\beta_{3}$ & $\beta_{4}$ & $\beta_{1}$ & $\beta_{2}$ & $\beta_{3}$ & $\beta_{4}$ \\
\hline \multirow[t]{4}{*}{0.5} & $P B_{I}$ & $\mathrm{i}$ & 98.78 & 98.33 & 98.23 & 98.60 & 95.40 & 95.05 & 95.57 & 95.18 \\
\hline & $P B_{D}$ & $\mathrm{i}$ & 98.53 & 97.38 & 98.19 & 98.33 & 89.02 & 90.31 & 90.93 & 87.96 \\
\hline & & ii & 97.03 & 92.60 & 95.99 & 96.54 & 82.38 & 82.79 & 84.95 & 81.47 \\
\hline & & iii & 99.49 & 98.52 & 99.14 & 99.58 & 86.38 & 89.05 & 89.09 & 85.73 \\
\hline \multirow[t]{4}{*}{0.75} & $P B_{I}$ & $\mathrm{i}$ & 98.83 & 98.46 & 98.18 & 98.82 & 93.50 & 94.51 & 94.47 & 94.14 \\
\hline & $P B_{D}$ & $\mathrm{i}$ & 98.43 & 97.49 & 98.07 & 98.76 & 87.79 & 90.07 & 89.86 & 87.82 \\
\hline & & ii & 97.25 & 91.18 & 97.03 & 97.60 & 82.77 & 82.12 & 85.96 & 82.52 \\
\hline & & iii & 99.51 & 98.72 & 99.01 & 99.60 & 85.04 & 88.59 & 88.11 & 84.76 \\
\hline \multirow[t]{4}{*}{0.90} & $P B_{I}$ & $\mathrm{i}$ & 98.93 & 97.92 & 97.98 & 99.02 & 90.03 & 92.58 & 92.11 & 91.31 \\
\hline & $P B_{D}$ & $\mathrm{i}$ & 98.32 & 96.28 & 98.02 & 98.76 & 84.87 & 87.58 & 87.22 & 85.28 \\
\hline & & ii & 96.56 & 89.46 & 97.85 & 98.36 & 80.06 & 80.03 & 85.00 & 80.98 \\
\hline & & iii & 99.18 & 97.78 & 98.91 & 99.68 & 82.23 & 86.52 & 85.66 & 82.09 \\
\hline
\end{tabular}

below 95 percent.

The results for the percentile method $C I_{P}$ from Table 5.4 and Table A.5 in the appendix show that bootstrap method $P B_{I}$ and weights i and iii using bootstrap method $P B_{D}$ all perform very well, with all confidence intervals having a coverage of at least 95 percent. Of these three weight i using $P B_{D}$ in general has the true coverage that is closest to the nominal 95 percent level. Weight ii using $P B_{D}$ performs considerably worse than the other three, with 11 of 48 confidence intervals in Table A.5 below 95 percent.

Finally, looking at the true coverages for the bias corrected percentile method $C I_{P B C}$ in Table 5.4 and Table A.6 in the appendix, it is found that all confidence intervals based on $P B_{D}$ perform badly. All these confidence intervals, for weights i, ii and iii, have true coverages below the nominal 95 percent. Even the confidence intervals for weight i based on the bootstrap method $P B_{I}$ perform badly, with only the confidence intervals for quantile $q=0.5$ in Table 5.4 having a true coverage of at least 95 percent.

As a conclusion we can say that confidence intervals based on $C I_{P}$ using weight iii for bootstrap method $P B_{D}$ and weight i using $P B_{D}$ and $P B_{I}$ perform well and can be recommended, as well as confidence intervals based on $C I_{N}$ using weight iii for $P B_{D}$ and weight i using $P B_{I}$. Possibly even weight i using $P B_{D}$ could be used for $C I_{N}$. However, the fact that the true coverage is at least as large as the nominal level is not the only thing that should be taken into consideration when choosing which method to use. The length of the confidence interval should be considered, as well. 
Table 5.5: Median length of bootstrap based confidence intervals for nominal 95 percent level, normal approximation and percentile methods, mean values taken over all $\rho, \mathrm{n}, \mathrm{T}$ and distributions.

\begin{tabular}{|c|c|c|c|c|c|c|c|c|c|c|}
\hline \multicolumn{3}{|c|}{ bootstrap } & \multicolumn{4}{|c|}{$C I_{N}$} & \multicolumn{4}{|c|}{$C I_{P}$} \\
\hline$q$ & method & weight & $\beta_{1}$ & $\beta_{2}$ & $\beta_{3}$ & $\beta_{4}$ & $\beta_{1}$ & $\beta_{2}$ & $\beta_{3}$ & $\beta_{4}$ \\
\hline \multirow[t]{4}{*}{0.5} & $P B_{I}$ & $\mathrm{i}$ & 6.14 & 2.74 & 2.05 & 0.36 & 5.22 & 2.47 & 1.85 & 0.38 \\
\hline & $P B_{D}$ & $\mathrm{i}$ & 3.59 & 1.90 & 1.32 & 0.27 & 3.22 & 1.79 & 1.23 & 0.28 \\
\hline & & ii & 2.87 & 1.64 & 1.06 & 0.24 & 2.61 & 1.57 & 1.00 & 0.24 \\
\hline & & iii & 3.58 & 2.07 & 1.36 & 0.28 & 3.14 & 1.90 & 1.24 & 0.29 \\
\hline \multirow[t]{4}{*}{0.75} & $P B_{I}$ & $\mathrm{i}$ & 8.16 & 4.04 & 2.75 & 0.46 & 6.87 & 3.29 & 2.50 & 0.48 \\
\hline & $P B_{D}$ & $\mathrm{i}$ & 5.02 & 2.78 & 1.88 & 0.35 & 4.39 & 2.47 & 1.73 & 0.36 \\
\hline & & ii & 3.45 & 1.96 & 1.28 & 0.28 & 3.07 & 1.85 & 1.19 & 0.29 \\
\hline & & iii & 4.88 & 3.02 & 1.89 & 0.36 & 4.12 & 2.61 & 1.69 & 0.37 \\
\hline \multirow[t]{4}{*}{0.90} & $P B_{I}$ & $\overline{\mathrm{i}}$ & 10.70 & 7.42 & 4.00 & 0.72 & 8.92 & 5.97 & 3.73 & 0.67 \\
\hline & $P B_{D}$ & $\mathrm{i}$ & 7.75 & 5.68 & 3.11 & 0.57 & 6.44 & 4.72 & 2.90 & 0.53 \\
\hline & & ii & 4.51 & 2.75 & 1.72 & 0.36 & 3.88 & 2.46 & 1.57 & 0.37 \\
\hline & & iii & 7.18 & 5.86 & 3.03 & 0.55 & 5.91 & 4.68 & 2.77 & 0.52 \\
\hline
\end{tabular}

Length of confidence intervals Table 5.5 gives median length of the confidence intervals using $C I_{N}$ and $C I_{P}$ for the different quantiles, $q=0.5, q=0.75$ and $q=0.9$, calculated as the mean values aggregated over the normal and lognormal distributions and all values of $\rho, n$ and T. Tables A.11 - A.12 in Appendix give more details, with the median length of the confidence intervals calculated as the mean only over $\rho$, thus giving values separately for the normal and lognormal distribution and the different choices of $n$ and $T$.

For both $C I_{N}$ and $C I_{P}$ the confidence intervals for weight i using $P B_{D}$ in general are considerably shorter than those for weight i using $P B_{I}$. When comparing weights $\mathrm{i}$ and iii for $P B_{D}$ it is found that for both $C I_{N}$ and $C I_{P}$ the confidence intervals for weight i are overall longer for $n=20, T=5$ and shorter for $n=50, T=10$, although for both cases the true coverage is generally somewhat larger for weight iii. Comparing $C I_{N}$ and $C I_{P}$ it is seen that the confidence intervals for $C I_{P}$ are overall somewhat shorter than those for $C I_{N}$, although the coverages are overall somewhat larger. The conclusion from these observations is that $C I_{P}$ with weight i using $P B_{D}$ should be used to get both good coverage and a short confidence interval.

Finally, one could mention something about the differences between the quantiles $q=0.5, q=0.75$ and $q=0.9$. As is seen from Tables 5.5 and A.11 - A.12, in general the confidence intervals for $q=0.9$ are longer than those for $q=0.75$, and the confidence intervals for $q=0.75$ are in turn longer than those for $q=0.5$. This is especially marked for the lognormal distribution.

\subsubsection{Comparison over the different sizes of correlation}

Table 5.6 gives the true coverage of 95 percent confidence intervals using $C I_{N}, C I_{N B C}, C I_{P}$ and $C I_{P B C}$ for the different quantiles, $q=0.5, q=0.75$ and $q=0.9$, calculated as the mean values over the normal and lognormal distributions and all values of $q, n$ and T. Tables A.7 - A.10 in the Appendix give more details, with the true coverages calculated as the mean only over $q$, thus giving results separately for the normal and lognormal distribution and the different choices of $n$ and $T$. In general, the performances for $C I_{N}, C I_{N B C}, C I_{P}$ and $C I_{P B C}$ seem to be roughly the same as for the quantiles $q=0.5, q=0.75$ and $q=0.9$ in Tables 5.4 and A.3 - A.6.

First, looking at the confidence intervals for the normal approximation method $C I_{N}$ in Tables 5.6 and A.7, it is seen that weight i using bootstrap method $P B_{I}$ and weight iii using bootstrap method $P B_{D}$ both have a true coverage of at least 95 percent for all confidence intervals. This is true also for weight i using $P B_{D}$ except for one case of $\beta_{4}$ when $\rho=0$ in Table A.7, where the true coverage is 94.8 
Table 5.6: Confidence intervals, true coverage in percent for nominal 95 percent level, normal approximation with and without bias correction together with percentile method with and without bias correction. Mean values taken over all q, n, T and distributions.

\begin{tabular}{|c|c|c|c|c|c|c|c|c|c|c|}
\hline \multicolumn{3}{|c|}{ bootstrap } & \multicolumn{4}{|c|}{$C I_{N}$} & \multicolumn{4}{|c|}{$C I_{N B C}$} \\
\hline$\rho$ & method & weight & $\beta_{1}$ & $\beta_{2}$ & $\beta_{3}$ & $\beta_{4}$ & $\beta_{1}$ & $\beta_{2}$ & $\beta_{3}$ & $\beta_{4}$ \\
\hline \multirow[t]{4}{*}{0} & $P B_{I}$ & i & 96.63 & 95.98 & 97.67 & 96.71 & 93.74 & 93.99 & 95.63 & 92.06 \\
\hline & $P B_{D}$ & $\mathrm{i}$ & 96.45 & 95.61 & 97.29 & 96.12 & 93.28 & 93.23 & 95.26 & 91.30 \\
\hline & & ii & 95.98 & 90.23 & 95.82 & 93.53 & 92.23 & 86.35 & 92.68 & 86.40 \\
\hline & & iii & 97.62 & 97.82 & 98.17 & 97.21 & 93.43 & 94.36 & 94.86 & 88.90 \\
\hline \multirow[t]{4}{*}{0.5} & $P B_{I}$ & $\mathrm{i}$ & 97.72 & 96.74 & 97.58 & 97.43 & 95.68 & 95.15 & 96.18 & $\overline{93.59}$ \\
\hline & $P B_{D}$ & $\mathrm{i}$ & 97.02 & 96.23 & 97.51 & 96.57 & 93.47 & 93.48 & 95.50 & 90.81 \\
\hline & & ii & 96.63 & 86.89 & 95.13 & 93.98 & 91.73 & 82.23 & 91.59 & 86.08 \\
\hline & & iii & 97.82 & 97.29 & 98.18 & 97.53 & 93.40 & 93.84 & 95.12 & 89.11 \\
\hline \multirow[t]{4}{*}{0.95} & $P B_{I}$ & $\mathrm{i}$ & 99.96 & 99.47 & 99.78 & 99.97 & 99.66 & 98.92 & 99.39 & 99.37 \\
\hline & $P B_{D}$ & $\mathrm{i}$ & 97.68 & 95.87 & 97.96 & 97.60 & 91.00 & 90.73 & 90.83 & 88.67 \\
\hline & & ii & 95.62 & 85.15 & 96.15 & 95.41 & 85.85 & 77.93 & 85.44 & 82.03 \\
\hline & & iii & 97.67 & 96.02 & 97.95 & 97.52 & 91.43 & 90.67 & 90.91 & 88.81 \\
\hline \multicolumn{3}{|c|}{ bootstrap } & \multicolumn{4}{|c|}{$C I_{P}$} & \multicolumn{4}{|c|}{$C I_{P B C}$} \\
\hline$\rho$ & method & weight & $\beta_{1}$ & $\beta_{2}$ & $\beta_{3}$ & $\beta_{4}$ & $\beta_{1}$ & $\beta_{2}$ & $\beta_{3}$ & $\beta_{4}$ \\
\hline \multirow[t]{4}{*}{0} & $P B_{I}$ & $\mathrm{i}$ & 98.25 & 97.58 & 97.61 & 98.16 & 89.21 & 91.15 & 91.43 & 89.71 \\
\hline & $P B_{D}$ & $\mathrm{i}$ & 97.89 & 96.68 & 97.37 & 97.85 & 88.83 & 89.91 & 90.58 & 88.81 \\
\hline & & ii & 96.17 & 92.98 & 96.02 & 96.21 & 83.68 & 84.15 & 87.03 & 83.32 \\
\hline & & iii & 99.68 & 99.11 & 99.02 & 99.55 & 83.23 & 86.77 & 87.23 & 83.53 \\
\hline \multirow[t]{4}{*}{0.5} & $P B_{I}$ & $\mathrm{i}$ & 98.44 & 97.45 & 96.95 & 98.29 & 91.62 & 92.54 & 91.99 & 91.82 \\
\hline & $P B_{D}$ & $\mathrm{i}$ & 98.38 & 97.08 & 97.53 & 98.26 & 87.63 & 89.73 & 90.23 & 88.00 \\
\hline & & ii & 97.08 & 90.78 & 95.67 & 96.68 & 82.75 & 81.64 & 86.49 & 83.23 \\
\hline & & iii & 99.52 & 98.43 & 98.60 & 99.52 & 85.05 & 88.64 & 88.29 & 84.63 \\
\hline \multirow[t]{4}{*}{0.95} & $P B_{I}$ & $\mathrm{i}$ & 99.85 & 99.68 & 99.83 & 99.98 & 98.11 & 98.44 & 98.72 & 99.11 \\
\hline & $P B_{D}$ & $\mathrm{i}$ & 99.00 & 97.38 & 99.38 & 99.73 & 85.21 & 88.31 & 87.20 & 84.25 \\
\hline & & ii & 97.59 & 89.47 & 99.19 & 99.61 & 78.78 & 79.14 & 82.38 & 78.41 \\
\hline & & iii & 98.98 & 97.48 & 99.44 & 99.79 & 85.38 & 88.75 & 87.34 & 84.43 \\
\hline
\end{tabular}

percent. Of these three, weight i using $P B_{D}$ overall has the true coverage that is closest to the nominal 95 percent. Weight ii again performs badly, with more than half of the confidence intervals in Table A.7 having true coverages of less than 95 percent.

For the bias corrected normal approximation method $C I_{N B C}$ it is from Tables 5.6 and A.8 found again that the confidence intervals based on $P B_{D}$ all perform badly, with almost all confidence intervals for weights i, ii and iii having true coverages that are less than 95 percent. Weight i using bootstrap method $P B_{I}$ performs somewhat better, but yet 4 of 12 confidence intervals in Table 5.6 and 20 of 48 confidence intervals in Table A.8 have a true coverage below 95 percent.

The true coverages for the percentile method $C I_{P}$, as given in Tables 5.6 and A.9, show that, as can be seen, all confidence intervals for weights i and iii using $P B_{D}$ have a true coverage of at least 95 percent, which also all but one of the confidence intervals for weight i using $P B_{I}$ in Table A.9 have. Of these three, again weight i using $P B_{D}$ in general has the true coverage that is closest to the nominal 95 percent level. Weight ii using $P B_{D}$ as usual performs considerably worse than the other three, with 3 of 12 confidence intervals in Table 5.6 and 9 of 48 confidence intervals in Table A.9 being below 95 percent.

Finally, Tables 5.6 and A.10 show that for the bias corrected percentile method $C I_{P B C}$ all confidence intervals for weight i using $P B_{I}$ have a true coverage percentage of at least 95 percent when $\rho=0.95$. Except for these cases, all confidence intervals for all weights and both $P B_{D}$ and $P B_{I}$ have true coverages that are less than the nominal level of 95 percent.

Thus one can draw the conclusion that confidence intervals based on $C I_{P}$ using weights i or iii for bootstrap method $P B_{D}$, and confidence intervals based on $C I_{N}$ using weights i for $P B_{I}$ or weight iii for 
Table 5.7: Median length of bootstrap based confidence intervals for nominal 95 percent level, normal approximation and percentile methods, mean values taken over all q, n, T and distributions.

\begin{tabular}{|c|c|c|c|c|c|c|c|c|c|c|}
\hline \multicolumn{3}{|c|}{ bootstrap } & \multicolumn{4}{|c|}{$C I_{N}$} & \multicolumn{4}{|c|}{$C I_{P}$} \\
\hline$\rho$ & method & weight & $\beta_{1}$ & $\beta_{2}$ & $\beta_{3}$ & $\beta_{4}$ & $\beta_{1}$ & $\beta_{2}$ & $\beta_{3}$ & $\beta_{4}$ \\
\hline \multirow[t]{4}{*}{0} & $P B_{I}$ & $\mathrm{i}$ & 8.42 & 4.98 & 2.99 & 0.55 & 7.04 & 4.15 & 2.77 & 0.52 \\
\hline & $P B_{D}$ & $\mathrm{i}$ & 7.92 & 4.70 & 2.87 & 0.55 & 6.67 & 3.92 & 2.64 & 0.50 \\
\hline & & ii & 4.89 & 2.58 & 1.74 & 0.37 & 4.15 & 2.30 & 1.57 & 0.37 \\
\hline & & iii & 7.73 & 5.22 & 2.92 & 0.55 & 6.21 & 4.16 & 2.58 & 0.52 \\
\hline \multirow[t]{4}{*}{0.5} & $P B_{I}$ & $\mathrm{i}$ & 8.54 & 4.94 & 3.06 & 0.53 & 7.22 & 4.02 & 2.82 & 0.52 \\
\hline & $P B_{D}$ & $\mathrm{i}$ & 6.32 & 3.97 & 2.52 & 0.45 & 5.25 & 3.42 & 2.30 & 0.45 \\
\hline & & ii & 4.02 & 2.28 & 1.54 & 0.34 & 3.50 & 2.12 & 1.42 & 0.34 \\
\hline & & iii & 5.80 & 4.05 & 2.43 & 0.45 & 4.84 & 3.39 & 2.20 & 0.45 \\
\hline \multirow[t]{4}{*}{0.95} & $P B_{I}$ & $\mathrm{i}$ & 8.05 & 4.29 & 2.75 & 0.46 & 6.75 & 3.56 & 2.50 & 0.49 \\
\hline & $P B_{D}$ & $\mathrm{i}$ & 2.12 & 1.68 & 0.93 & 0.20 & 2.12 & 1.64 & 0.92 & 0.21 \\
\hline & & ii & 1.91 & 1.49 & 0.78 & 0.18 & 1.91 & 1.45 & 0.77 & 0.19 \\
\hline & & iii & 2.11 & 1.68 & 0.93 & 0.20 & 2.12 & 1.64 & 0.92 & 0.21 \\
\hline
\end{tabular}

$P B_{D}$ perform well and can be recommended. Possibly could even weight i using $P B_{I}$ be used for $C I_{P}$ and weight i using $P B_{D}$ be used for $C I_{N}$.

Length of confidence intervals Now, let us look at the median length of the confidence intervals using $C I_{N}$ and $C I_{P}$ for the different sizes of correlation, $\rho=0, \rho=0.5$ and $\rho=0.95$. These are given in Table 5.7, calculated as the mean values over the normal and lognormal distributions and all values of $\rho, n$ and $T$. Tables A.13 - A.14 in Appendix give more details, with the median length of the confidence intervals calculated separately, as the mean values over $q$, for the normal and lognormal distribution and the different choices of $n$ and $T$.

Again the results are roughly the same as for the quantiles $q=0.5, q=0.75$ and $q=0.9$ in Tables 5.5 and A.11 - A.12. For both $C I_{N}$ and $C I_{P}$, the confidence intervals for weight i using $P B_{D}$ are overall shorter than those for weight i using $P B_{I}$, and the confidence intervals for weight i using $P B_{D}$ are overall longer than those for weight iii using $P B_{D}$ for $n=20, T=5$ and shorter for $n=50, T=10$, although for both cases the true coverage is generally somewhat larger for weight iii. Comparing $C I_{N}$ and $C I_{P}$ it is again seen that, overall, the confidence intervals for $C I_{P}$ are somewhat shorter than those for $C I_{N}$, the true coverage is generally somewhat larger. The conclusion from these observations is, as before, that $C I_{P}$ with weight i using $P B_{D}$ should be used to get both good coverage and a short confidence interval.

Let us also finally say something about the differences between the correlation sizes, $\rho=0, \rho=0.5$ and $\rho=0.95$. Tables 5.7 and A.13 - A.14 give that, overall, an increasing correlation leads to shorter confidence intervals. Thus, overall, the confidence intervals for $\rho=0.95$ are shorter than those for $\rho=0.5$, which in turn are shorter than those for $\rho=0$. Again, this is especially marked for the lognormal distribution.

\section{Application to the growth patterns of two genotypes of soy- bean}

A study aimed at comparing the growth patterns of two genotypes of soybean was performed 1988-1990 at the Department of Crop Science at North Carolina State University. The data from this study is presented and analyzed in Davidian and Giltinan (1993). Of the two genotypes of soybean was one an experimental strain, called Plant Introduction No. $416937(P)$, while the other one was a commercial 
Table 5.8: Parameter estimates for mean and quantile regression of the commercial soybean variety Forrest (F), unweighted estimates without and with bias correction

\begin{tabular}{c|rrr|rrr|} 
& \multicolumn{3}{|c|}{ not bias corrected } & \multicolumn{3}{c|}{ bias corrected } \\
$q$ & \multicolumn{1}{|c}{$\beta_{1}$} & $\beta_{2}$ & \multicolumn{1}{c}{$\beta_{3}$} & \multicolumn{1}{c}{$\beta_{1}$} & $\beta_{2}$ & $\beta_{3}$ \\
\hline 0.1 & 9.73 & 51.96 & -0.145 & 9.55 & 51.27 & -0.150 \\
0.2 & 11.21 & 52.12 & -0.141 & 11.14 & 52.22 & -0.140 \\
0.3 & 11.83 & 50.87 & -0.145 & 10.75 & 49.43 & -0.151 \\
0.4 & 14.61 & 53.10 & -0.134 & 13.77 & 52.39 & -0.137 \\
0.5 & 17.74 & 54.98 & -0.124 & 17.88 & 54.97 & -0.124 \\
0.6 & 19.10 & 55.55 & -0.120 & 19.18 & 55.55 & -0.121 \\
0.7 & 20.29 & 55.77 & -0.113 & 20.59 & 56.05 & -0.111 \\
0.8 & 20.65 & 54.42 & -0.118 & 20.69 & 54.38 & -0.119 \\
0.9 & 20.95 & 53.41 & -0.116 & 20.48 & 53.02 & -0.116 \\
\hline mean & 16.04 & 53.67 & -0.129 & 15.97 & 53.57 & -0.130 \\
\hline
\end{tabular}

Table 5.9: Parameter estimates for mean and quantile regression for the experimental soybean strain Plant Introduction No. 416937 (P), unweighted estimates without and with bias correction

\begin{tabular}{c|ccc|ccc|} 
& \multicolumn{3}{|c|}{ not bias corrected } & \multicolumn{3}{|c|}{ bias corrected } \\
\hline & $\beta_{1}$ & $\beta_{2}$ & $\beta_{3}$ & $\beta_{1}$ & $\beta_{2}$ & $\beta_{3}$ \\
\hline 0.1 & 16.97 & 55.66 & -0.121 & 16.83 & 55.91 & -0.121 \\
0.2 & 17.65 & 54.16 & -0.122 & 17.40 & 53.68 & -0.123 \\
0.3 & 18.50 & 54.43 & -0.118 & 18.55 & 54.66 & -0.117 \\
0.4 & 18.61 & 53.33 & -0.121 & 18.39 & 53.09 & -0.122 \\
0.5 & 19.51 & 52.98 & -0.124 & 19.33 & 52.68 & -0.125 \\
0.6 & 21.15 & 53.84 & -0.119 & 21.08 & 53.76 & -0.120 \\
0.7 & 22.87 & 54.66 & -0.114 & 23.12 & 54.85 & -0.114 \\
0.8 & 23.53 & 54.57 & -0.110 & 23.46 & 54.89 & -0.107 \\
0.9 & 24.02 & 52.97 & -0.114 & 22.01 & 51.57 & -0.118 \\
\hline mean & 20.82 & 54.36 & -0.116 & 20.78 & 54.31 & -0.116 \\
\hline
\end{tabular}

variety, called Forrest $(F)$.

The study was conducted as follows: For each of the three years 1988-1990 were 8 plots planted with seeds from soybean $P$ and 8 with seeds from soybean $F$, thus giving a total of 16 plots. Each of these plots was then sampled 8-10 times with approximately weekly intervals, with the first sample taken at day 14 or 15 and the last sample taken between 63 and 70 days later. This means that the data set consists of 16 subjects for each year, thus with a total of 48 subjects, each having 8-10 observations. Of these 48 subjects 24 belong to each of the two genotypes.

At each of the sampling times 6 plants were selected at random from each of the 16 plots, and the leaves from these plants were then aggregated and weighted separately for each plot to determine the average leaf weight, in grams, per plant in the plot. This leaf weight was then used to assess the growth of the soybeans. For further details about the data and the experiment, see Davidian and Giltinan (1993) and Chapters 1 and 11 in Davidian and Giltinan (1995).

\subsection{Model and methodology}

Besides being analyzed by Davidian and Giltinan (1993), with the same analysis used later by Davidian and Giltinan (1995), this data has also been analyzed by Pinheiro and Bates (2000) and Karlsson (2005), all using a three-parameter logistic growth function. Both Davidian and Giltinan (1993) and Pinheiro and Bates (2000) used mean regression models, while Karlsson (2005) used a quantile regression model.

The model with a three-parameter logistic growth function which Davidian and Giltinan (1993) and Karlsson (2005) used can for the marginal analysis approach (2.1) of this paper be written as 


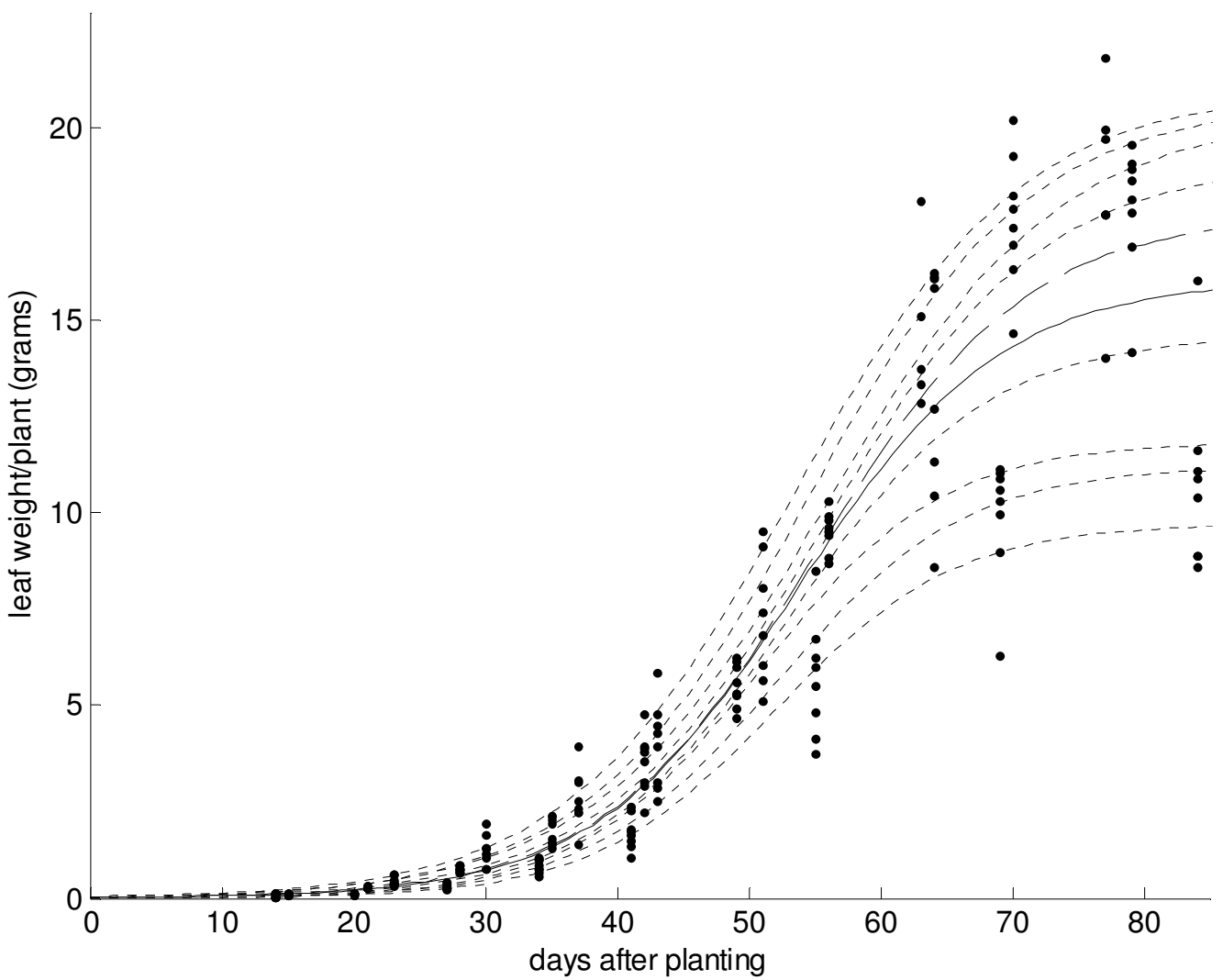

Figure 6.1: Unweighted mean and quantile regression lines for soybean Forrest, without bias correction. Mean regression line is solid, quantile regression lines $(q=0.1, \ldots, 0.4,0.6, \ldots, 0.9)$ are dotted, with median regression line dashed.

$$
y_{i t}=\frac{\beta_{1}}{1+\exp \left(\beta_{3}\left(x_{i t}-\beta_{2}\right)\right)}+\varepsilon_{i t}, \quad \beta_{1}, \beta_{2}>0, \beta_{3}<0, i=1, \ldots, n, t=1, \ldots, T_{i},
$$

where $x_{i t}$ is the day when sample number $t$ was taken for plant $i$. In this function is $\beta_{1}$ gives the asymptotic leaf weight per plant, $\beta_{2}$ the $E C_{50}$ value, i.e., the day at which half the asymptotic leaf weight per plant is achieved while parameter $\beta_{3}$ is a constant that governs the steepness of the growth curve.

In the following analysis, (6.1) will be used to separately estimate the growth pattern for the two genotypes of soybean $P$ and $F$, aggregated over all three years. This means that $n=24$ and $T_{i}=8,9$ or 10 in (6.1). In the analysis, the unweighted quantile regression estimator

$$
\widehat{\boldsymbol{\beta}}(q)=\min _{\boldsymbol{\beta}} \sum_{i=1}^{n} \sum_{t=1}^{T_{i}} \rho_{q}\left(y_{i t}-f\left(\mathbf{x}_{i t}, \boldsymbol{\beta}\right)\right),
$$

will be used to estimate nine quantiles, $q=0.1,0.2, \ldots, 0.9$.

On the basis of the performances for both bias correction and confidence interval calculation, the following methods will then be used for the quantile regression estimates from (6.2): They will be bias corrected using the $C I_{N B C}$ method (3.5). Finally, the block bootstrap method $P B_{D}$ will be used to calculate 95 percent confidence intervals using the percentile method $C I_{P}$ and the normal approximation method $C I_{N}$. The number of bootstrap resamplings $B$ that will be used will be set to $B=1000$. The 


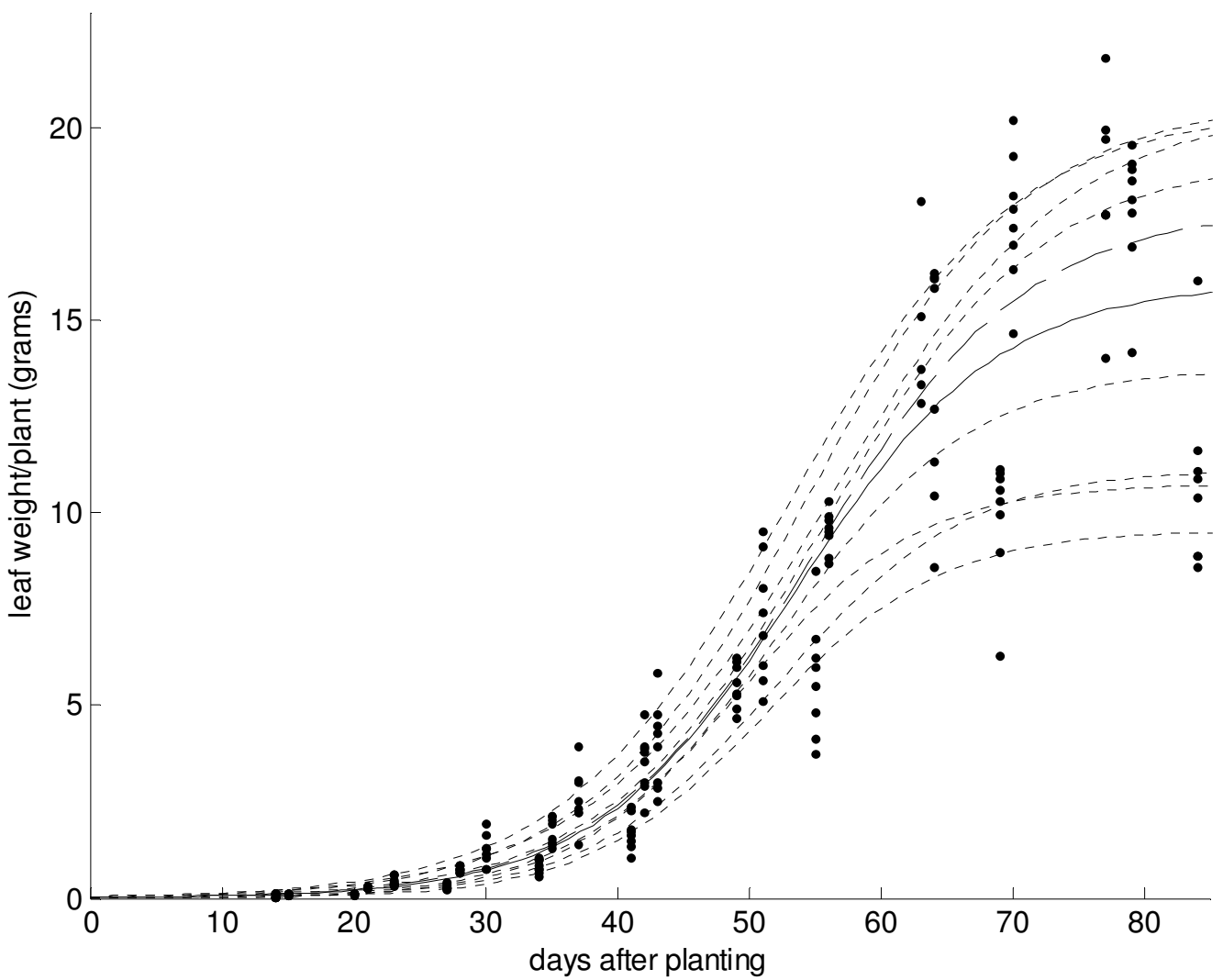

Figure 6.2: Unweighted mean and quantile regression lines for soybean Forrest, with bias correction. Mean regression line is solid, quantile regression lines $(q=0.1, \ldots, 0.4,0.6, \ldots, 0.9)$ are dotted, with median regression line dashed.

weights used will be the unweighted alternative, weight $\mathrm{i}$, with $w_{i t}=1$. Note that the alternative $\mathbf{W}_{i}=$ $\left(1 /\left|s_{t t^{\prime}}\right|\right)$, weight iii, can not be used, since $T_{i}$ is not equal for all subjects. i.e., all subjects are not measured equally many times.

\subsection{Analysis}

Tables 5.8 and 5.9 give the parameter estimates of the nine quantiles $q=0.1,0.2, \ldots, 0.9$ and the mean regression for the commercial variety Forrest $(F)$ and the experimental strain Plant Introduction No. $416937(P)$, respectively. They also give the bias corrected parameter estimates using bootstrap method $P B_{D}$.

Figure 6.1 show, for soybean type $F$, the mean (solid) and quantile (dotted, with median dashed) regression curves for the quantiles $q=0.1,0.2, \ldots, 0.9$, using the non bias corrected estimates from Table 5.8 , together with the original data points, while Figure 6.2 show the same thing using the bias corrected estimates from Table 5.8. Tables 6.1 - 6.4 give 95 percent confidence intervals using the percentile and normal approximation methods with bootstrap method $P B_{D}$.

First, comparing the original parameter estimates with the bias corrected parameter estimates it is seen that there are sometimes quite large changes. There are two main problems with the bias corrected parameter estimates. Since the parameter $\beta_{1}$ gives the asymptotic leaf weight per plant, increasing the quantile values $q$ should also lead to increasing values of the parameter $\beta_{1}$, so that two quantile regression curves never cross. This is true for the original estimates for both Forrest $(F)$ and Plant Introduction 
Table 6.1: 95 per cent confidence intervals for mean and quantile regression of the commercial soybean variety Forrest $(\mathrm{F})$, bootstrap percentile method without bias correction using unweighted estimates

\begin{tabular}{c|rrr|ccc|} 
& \multicolumn{3}{|c|}{ lower limit for CI } & \multicolumn{3}{c|}{ upper limit for CI } \\
$q$ & $\beta_{1}$ & $\beta_{2}$ & $\beta_{3}$ & $\beta_{1}$ & $\beta_{2}$ & $\beta_{3}$ \\
\hline 0.1 & 8.71 & 49.52 & -0.163 & 11.46 & 56.60 & -0.122 \\
0.2 & 8.98 & 49.65 & -0.156 & 14.79 & 55.12 & -0.124 \\
0.3 & 10.87 & 49.75 & -0.155 & 18.09 & 56.49 & -0.118 \\
0.4 & 11.41 & 49.67 & -0.152 & 19.29 & 57.22 & -0.116 \\
0.5 & 11.77 & 50.34 & -0.147 & 19.83 & 56.92 & -0.114 \\
0.6 & 16.28 & 53.33 & -0.131 & 20.56 & 56.83 & -0.110 \\
0.7 & 18.70 & 53.30 & -0.127 & 21.67 & 57.27 & -0.106 \\
0.8 & 19.00 & 51.86 & -0.129 & 22.41 & 57.53 & -0.105 \\
0.9 & 19.69 & 51.60 & -0.127 & 24.45 & 57.62 & -0.105 \\
\hline mean & 14.21 & 52.47 & -0.143 & 18.17 & 55.21 & -0.115 \\
\hline
\end{tabular}

Table 6.2: 95 per cent confidence intervals for mean and quantile regression of the commercial soybean variety Forrest $(\mathrm{F})$, bootstrap normal approximation method without bias correction using unweighted estimates

\begin{tabular}{c|rrr|ccc|} 
& \multicolumn{3}{|c|}{ lower limit for CI } & \multicolumn{3}{c}{ upper limit for CI } \\
$q$ & \multicolumn{1}{|c}{$\beta_{1}$} & $\beta_{2}$ & $\beta_{3}$ & $\beta_{1}$ & $\beta_{2}$ & $\beta_{3}$ \\
\hline 0.1 & 7.79 & 48.44 & -0.165 & 11.67 & 55.47 & -0.126 \\
0.2 & 8.90 & 49.47 & -0.156 & 13.53 & 54.76 & -0.125 \\
0.3 & 7.79 & 47.22 & -0.164 & 15.88 & 54.52 & -0.126 \\
0.4 & 9.59 & 48.88 & -0.155 & 19.64 & 57.32 & -0.113 \\
0.5 & 13.70 & 51.82 & -0.140 & 21.77 & 58.14 & -0.109 \\
0.6 & 16.72 & 53.72 & -0.131 & 21.48 & 57.37 & -0.110 \\
0.7 & 18.58 & 53.80 & -0.124 & 22.01 & 57.74 & -0.103 \\
0.8 & 18.84 & 51.72 & -0.130 & 22.46 & 57.12 & -0.106 \\
0.9 & 18.59 & 50.91 & -0.126 & 23.32 & 55.92 & -0.106 \\
\hline mean & 14.09 & 52.28 & -0.143 & 17.99 & 55.06 & -0.116 \\
\hline
\end{tabular}

$(P)$, as can be seen from Figure 6.1 for Forrest, but not for the bias corrected parameter estimates. For Forrest, both quantiles $q=0.3$ and $q=0.9$ in Table 5.8 have lower values than the preceding quantile, leading to crossing quantile regression curves, as can be seen from Figure 6.1. From Table 5.9 it can be seen that this is also the case for quantiles $q=0.4$ and $q=0.9$ for Plant Introduction. This is the first problem.

The second problem with the bias corrected parameter estimates is that, when using them together with the confidence intervals using the percentile method $C I_{P}$ in Tables 6.1 and 6.3, they are for several cases outside the confidence intervals. For example, the bias corrected parameter estimates for $\beta_{1}$ are below the lower confidence limits for both quantile $q=0.3$ for Forrest and quantile $q=0.9$ for Plant Introduction. Thus, the bias corrected parameter estimates could hardly be used together with the percentile method $C I_{P}$ for this case. Note, however, that this problem does not arise when using the normal approximation method $C I_{N}$ in Tables 6.2 and 6.4 instead.

Comparing the original parameter estimates for the commercial variety Forrest and the experimental strain Plant Introduction No. 416937 it is seen that $\widehat{\beta}_{1}(0.6)=16.28$ for Forrest and $\widehat{\beta}_{1}(0.1)=16.97$ for Plant Introduction. Thus we can conclude that 90 percent of the soybean plants from Plant Introduction have an asymptotic leaf weight that is larger than 60 percent of the soybean plants from Forrest. The values $\widehat{\beta}_{1}(0.9)=19.69$ for $\mathrm{F}$ and $\widehat{\beta}_{1}(0.6)=21.15$ for $P$ tells us that 40 percent of the soybean plants from Plant Introduction have an asymptotic leaf weight that is larger than 90 percent of the soybean plants from Forrest. 
Table 6.3: 95 per cent confidence intervals for mean and quantile regression of the experimental soybean strain Plant Introduction No. 416937 (P), bootstrap percentile method without bias correction using unweighted estimates

\begin{tabular}{c|ccc|ccc|} 
& \multicolumn{3}{|c|}{ lower limit for CI } & \multicolumn{3}{c|}{ upper limit for CI } \\
$q$ & $\beta_{1}$ & $\beta_{2}$ & $\beta_{3}$ & $\beta_{1}$ & $\beta_{2}$ & $\beta_{3}$ \\
\hline 0.1 & 15.87 & 52.48 & -0.130 & 18.43 & 57.50 & -0.113 \\
0.2 & 16.51 & 52.52 & -0.127 & 19.24 & 57.40 & -0.112 \\
0.3 & 17.53 & 52.22 & -0.128 & 19.51 & 56.01 & -0.113 \\
0.4 & 17.72 & 51.57 & -0.131 & 20.64 & 55.99 & -0.111 \\
0.5 & 18.30 & 51.14 & -0.132 & 22.47 & 56.55 & -0.109 \\
0.6 & 18.46 & 51.26 & -0.131 & 23.85 & 57.00 & -0.106 \\
0.7 & 20.16 & 51.79 & -0.126 & 23.97 & 56.54 & -0.106 \\
0.8 & 21.67 & 51.43 & -0.123 & 25.36 & 56.60 & -0.105 \\
0.9 & 22.69 & 50.78 & -0.124 & 35.31 & 62.37 & -0.091 \\
\hline mean & 19.34 & 52.50 & -0.126 & 22.51 & 56.29 & -0.106 \\
\hline
\end{tabular}

Table 6.4: 95 per cent confidence intervals for mean and quantile regression of the experimental soybean strain Plant Introduction No. 416937 (P), bootstrap normal approximation method without bias correction using unweighted estimates

\begin{tabular}{c|ccc|ccc|} 
& \multicolumn{2}{|c|}{ lower limit for CI } & \multicolumn{3}{c|}{ upper limit for CI } \\
$q$ & $\beta_{1}$ & $\beta_{2}$ & $\beta_{3}$ & $\beta_{1}$ & $\beta_{2}$ & $\beta_{3}$ \\
\hline 0.1 & 15.43 & 53.09 & -0.130 & 18.51 & 58.23 & -0.112 \\
0.2 & 16.20 & 51.87 & -0.129 & 19.09 & 56.46 & -0.114 \\
0.3 & 17.47 & 52.48 & -0.126 & 19.54 & 56.38 & -0.110 \\
0.4 & 17.26 & 51.15 & -0.131 & 19.95 & 55.52 & -0.112 \\
0.5 & 17.24 & 50.27 & -0.135 & 21.77 & 55.68 & -0.112 \\
0.6 & 18.40 & 50.96 & -0.131 & 23.90 & 56.72 & -0.107 \\
0.7 & 20.85 & 52.35 & -0.124 & 24.89 & 56.97 & -0.105 \\
0.8 & 20.83 & 51.56 & -0.120 & 26.22 & 57.57 & -0.100 \\
0.9 & 16.12 & 46.64 & -0.131 & 31.92 & 59.29 & -0.097 \\
\hline mean & 19.25 & 52.50 & -0.126 & 22.40 & 56.21 & -0.106 \\
\hline
\end{tabular}

\section{Summary and conclusions}

This paper has examined the use of bootstrapping for reducing bias and calculating confidence intervals for a weighted nonlinear quantile regression estimator for longitudinal data using simulation methods. Two different paired bootstrap methods have been used, one where the dependence of the data is disregarded and the bootstrap resampling is performed as if all observations for all subjects where independent, $P B_{I}$, and one where the dependence of the data forms the basis of the resampling, $P B_{D}$. For the latter, the bootstrap is performed by resampling the pairs $\left(\mathbf{y}_{i}, \mathbf{X}_{i}\right)$ of dependent and independent variables consisting of all observations for a subject.

Three different weights were considered for the weighted nonlinear quantile regression estimator: the unweighted alternative weight i, using the same weight for all time points and subjects, weight ii, which weights the observations with regard to the dispersions over the time points for the individual subjects, and weight iii, that weights the observations with regard to the dispersions over both time points and subjects. Of these where all three used with $P B_{D}$ but only weight i with $P B_{I}$, since the other weights could not be used with this bootstrap method.

For the bootstrap based bias correction it was found that bootstrap method $P B_{I}$ performed badly in improving bias, in fact, overall the bias deteriorated when using $P B_{I}$. However, the bootstrap method $P B_{D}$ performed well, with overall somewhat improving biases for all weights used. Overall, weight iii had the lowest bias, followed by the unweighted alternative weight i. It is also found that, overall, the bias improvements increases with values of $q$ further away from the median, and that the bias improvements 
decreases with increasing $\rho$.

Regarding the bootstrap based confidence interval calculations, four alternatives were used: the normal approximation method $C I_{N}$, the bias corrected normal approximation method $C I_{N B C}$, the percentile method $C I_{P}$ and the bias corrected percentile method $C I_{P B C}$. These were compared for calculations of 95 percent confidence intervals. The bias corrected methods $C I_{N B C}$ and $C I_{P B C}$ performed bad for all weights and both bootstrap methods $P B_{I}$ and $P B_{D}$, with true coverage percentages usually below the nominal 95 percent level. But weight i, using $P B_{I}$ or $P B_{D}$, and weight iii for $P B_{D}$, performed well for both the normal approximation method $C I_{N}$ and the percentile method $C I_{P}$, with all or nearly all confidence intervals having a true coverage of at least the nominal 95 percent level. Overall, weight i using $P B_{D}$ for percentile method $C I_{P}$ performed best in terms of coverage and length of confidence interval, followed by weight iii for percentile method $C I_{P}$. It is also found that values of $q$ further away from the median leads to longer confidence intervals, while increasing the correlation $\rho$ leads to shorter confidence intervals.

The use of bootstrapping for reducing bias and calculating confidence intervals for a weighted nonlinear quantile regression estimator is also applied to a real longitudinal data set with growth patterns, measured as leaf weight per plant, of two genotypes of soybean, where nonlinear quantile regressions are calculated for the nine quantiles $q=0.1,0.2, \ldots, 0.9$. For this application is weight i used with bootstrap method $P B_{D}$, and confidence intervals calculated using the percentile method $C I_{P}$ and the normal approximation method $C I_{N}$. It is found that using bias correction leads to some problems for this case. One problem is that when using bias corrected parameter estimates for the quantile regression one sometimes get crossing quantile regression curves. Another problem is that the bias corrected parameter estimates sometimes are outside the limits of the confidence intervals for the percentile method $C I_{P}$.

As a conclusion, with regards to the performance for both bias correction and confidence interval calculation, the following recommendations can be issued when performing nonlinear quantile regression estimation for longitudinal data: use either weight $\mathrm{i}$ or weight iii, without bias correction, and calculate the confidence intervals with the percentile method $C I_{P}$ using the bootstrap method $P B_{D}$. 


\section{References}

Buchinsky, M. (1994). Changes in the U.S. wage structure 1963-1987: Application of quantile regression. Econometrica 62 (2), 405-458.

Buchinsky, M. (1995). Estimating the asymptotic covariance matrix for quantile regression models: A Monte Carlo study. Journal of Econometrics 68, 303-338.

Buchinsky, M. (1998). Recent Advances in Quantile Regression Models: A Practical Guideline for Empirical Research. The Journal of Human Resources 33, 88-126.

Carpenter, J. And Bithell, J. (2000) . Bootstrap confidence intervals: when, which, what? A practical guide for medical statisticians. Statistics in Medicine 19, 1141-1164.

Davidian, M. And GiLtinan, D. M. (1993). Some general estimation methods for nonlinear mixedeffects models. Journal of Biopharmaceutical Statistics 3, 23-55.

Davidian, M. And Giltinan, D. M. (1995). Nonlinear Models for Repeated Measurement Data. Chapman \& Hall, London.

Davis, C. S. (2002). Statistical Methods for the analysis of Repeated Measurements. Springer, Berlin.

Davison, A. C. And Hinkley, D. V. (1997). Bootstrap Methods and their Application. Cambridge University Press, Cambridge.

Diggle, P. J., Heagerty, P., Liang, K-Y and Zeger, S. L. (2002). Analysis of Longitudinal Data. Oxford University Press, Oxford.

Efron, B. (1979). Bootstrap Methods: Another Look at the Jackknife. Annals of Statistics 7 (1), 1-26. Fitzenberger, B. (1997).The moving blocks bootstrap and robust inference for linear least squares and quantile regressions. Journal of Econometrics 82, 253-287.

Hahn, J. (1995). Bootstrapping quantile regression estimators. Econometric Theory 11, 105-121.

HE, X. AND KIM, M-O (2002). On marginal estimation in a semiparametric model for longitudinal data with time-independent covariates. Metrika, 55, 67-74.

He, X., Fu, B. And Fung, W. K. (2003). Median Regression for Longitudinal Data. Statistics in Medicine 22 (23), 3655-3669.

Horowitz, J. L. (1998). Bootstrap methods for median regression methods. Econometrica 66 (6), 1327-1351.

Jung, S-H (1996). Quasi-Likelihood for Median Regression Models. Journal of the American Statistical Association 91, 251-257.

KarLSson, A. (2005). Nonlinear Quantile Regression Estimation of Longitudinal Data. Unpublished manuscript, Division of Statistics, Department of Information Science, Uppsala University.

Koenker, R. (2004). Quantile regression for longitudinal data. Journal of Multivariate Analysis 91, $74-89$.

Koenker, R. (2005). Quantile Regression. Cambridge University Press, Cambridge.

Koenker, R. and Bassett, G. (1978). Regression Quantiles. Econometrica 46 (1), 33-50.

LahiRi, S. N. (2003). Resampling Methods for Dependent Data. Springer, Berlin.

Lipsitz, S. R., Fitzmaurice, G. M., Molenberghs G. and Zhao, L. P. (1997). Quantile Regression Methods for Longitudinal Data with Drop-outs: Application to CD4 Cell Counts of Patients Infected with the Human Immunodeficiency Virus. Applied Statistics 46 (4), 463-476.

Manly, B. F. J. (1997). Randomization, Bootstrap and Monte Carlo Methods in Biology, 2nd ed. Chapman \& Hall, London.

Oberhofer, W. AND HAupt, H. (2005). Nonlinear quantile regression under dependence and heterogeneity. University of Regensburg Discussion Papers in Economics 388.

Pinheiro, J. C. and Bates, D. M. (2000). Mixed-Effects Models in S and S-PLUS. Springer, Berlin. Shao, J. And Tu, D. (1995). The Jackknife and Bootstrap. Springer, Berlin. 
Vonesh, E. F. AND Chinchilli, V. M. (1997). Linear and nonlinear models for the analysis of repeated measurements. Marcel Dekker, New York.

Yu, K. (2004). Nonparametric quantile regression for longitudinal data analysis. Unpublished manuscript, School of Mathematics and Statistics, University of Plymouth.

Yu, K., Lu, Z. AND Stander, J. (2003). Quantile regression: applications and current research areas.

The Statistician $\mathbf{5 2}$ (3), 331-350. 


\section{A Appendix}

Table A.1: Improvement in bias, in percentage points, using bias correction, mean values over all values of $\rho$

\begin{tabular}{|c|c|c|c|c|c|c|c|c|c|c|c|}
\hline distr. & $q$ & $\begin{array}{l}\text { bootstrap } \\
\text { method }\end{array}$ & weight & $\beta_{1}$ & $\begin{array}{c}n=20 \\
\beta_{2}\end{array}$ & $\begin{array}{c}T=5 \\
\beta_{3}\end{array}$ & $\beta_{4}$ & $\beta_{1}$ & $\begin{array}{c}\imath=50 \\
\beta_{2}\end{array}$ & $\begin{array}{c}T=10 \\
\beta_{3}\end{array}$ & $\beta_{4}$ \\
\hline \multirow[t]{12}{*}{ normal } & 0.5 & $P B_{I}$ & $\mathrm{i}$ & 0.57 & 0.25 & -0.01 & -0.28 & 0.02 & 0.19 & -0.13 & -0.03 \\
\hline & & $P B_{D}$ & $\mathrm{i}$ & 2.06 & 0.61 & 0.40 & 0.06 & 0.30 & 0.23 & -0.11 & 0.01 \\
\hline & & & ii & 0.27 & 0.14 & -0.26 & 1.09 & 0.69 & 0.22 & -0.05 & -0.06 \\
\hline & & & iii & 1.34 & 0.53 & -0.05 & 0.69 & 0.43 & 0.24 & 0.01 & 0.17 \\
\hline & 0.75 & $P B_{I}$ & $\mathrm{i}$ & 0.51 & 0.26 & -0.27 & 0.00 & -0.14 & 0.07 & -0.20 & 0.20 \\
\hline & & $P B_{D}$ & $\mathrm{i}$ & 2.23 & 0.67 & 0.21 & 1.21 & 0.20 & 0.14 & -0.05 & 0.25 \\
\hline & & & ii & 1.22 & 0.16 & -0.01 & 1.16 & 0.88 & -0.07 & 0.07 & 0.17 \\
\hline & & & iii & 0.64 & 0.45 & -0.86 & 1.45 & 0.56 & 0.25 & 0.04 & 0.18 \\
\hline & 0.90 & $P B_{I}$ & $\mathrm{i}$ & -0.15 & -0.07 & -1.30 & 1.24 & -0.01 & 0.07 & -0.15 & 0.54 \\
\hline & & $P B_{D}$ & $\mathrm{i}$ & 0.78 & 0.60 & -1.06 & 2.65 & 0.51 & 0.19 & 0.15 & 0.53 \\
\hline & & & ii & 0.52 & -0.04 & -0.91 & 1.47 & 1.28 & -0.29 & 0.14 & 0.48 \\
\hline & & & iii & 0.65 & 0.63 & -1.23 & 2.31 & 0.86 & 0.20 & 0.24 & 0.83 \\
\hline \multirow[t]{12}{*}{ lognormal } & 0.5 & $P B_{I}$ & $\mathrm{i}$ & -2.36 & -0.25 & -1.13 & -0.03 & -0.01 & 0.05 & -0.07 & -0.06 \\
\hline & & $P B_{D}$ & $\mathrm{i}$ & 0.50 & 0.23 & 0.23 & -0.12 & 0.10 & 0.08 & -0.01 & -0.12 \\
\hline & & & ii & 0.32 & -0.04 & 0.17 & -0.04 & 0.07 & -0.06 & -0.01 & 0.18 \\
\hline & & & iii & 0.37 & 0.23 & 0.10 & 0.29 & 0.05 & 0.08 & -0.05 & 0.03 \\
\hline & 0.75 & $P B_{I}$ & $\mathrm{i}$ & -0.77 & -0.79 & -0.13 & -1.63 & -0.42 & -0.02 & -0.22 & 0.11 \\
\hline & & $P B_{D}$ & $\mathrm{i}$ & 1.05 & 0.06 & 0.19 & -0.57 & -0.09 & 0.08 & -0.14 & 0.15 \\
\hline & & & ii & 0.49 & -0.21 & 0.15 & 0.75 & 0.35 & -0.16 & -0.08 & 0.18 \\
\hline & & & iii & 0.81 & 0.63 & -0.81 & 1.05 & 0.21 & 0.14 & -0.12 & 0.24 \\
\hline & 0.90 & $P B_{I}$ & $\mathrm{i}$ & -0.85 & 0.89 & -4.11 & 0.86 & 1.13 & 0.51 & 0.30 & 1.78 \\
\hline & & $P B_{D}$ & $\mathrm{i}$ & 0.82 & 2.20 & -4.09 & 5.84 & 2.02 & 0.84 & 0.30 & 1.36 \\
\hline & & & ii & 0.61 & -0.46 & -0.39 & 1.43 & 1.17 & -0.43 & -0.18 & 0.35 \\
\hline & & & iii & 0.46 & 1.68 & -0.12 & 4.43 & 2.14 & 1.03 & -0.51 & 2.05 \\
\hline
\end{tabular}


Table A.2: Improvement in bias, in percentage points, using bias correction, mean values over all quantiles q

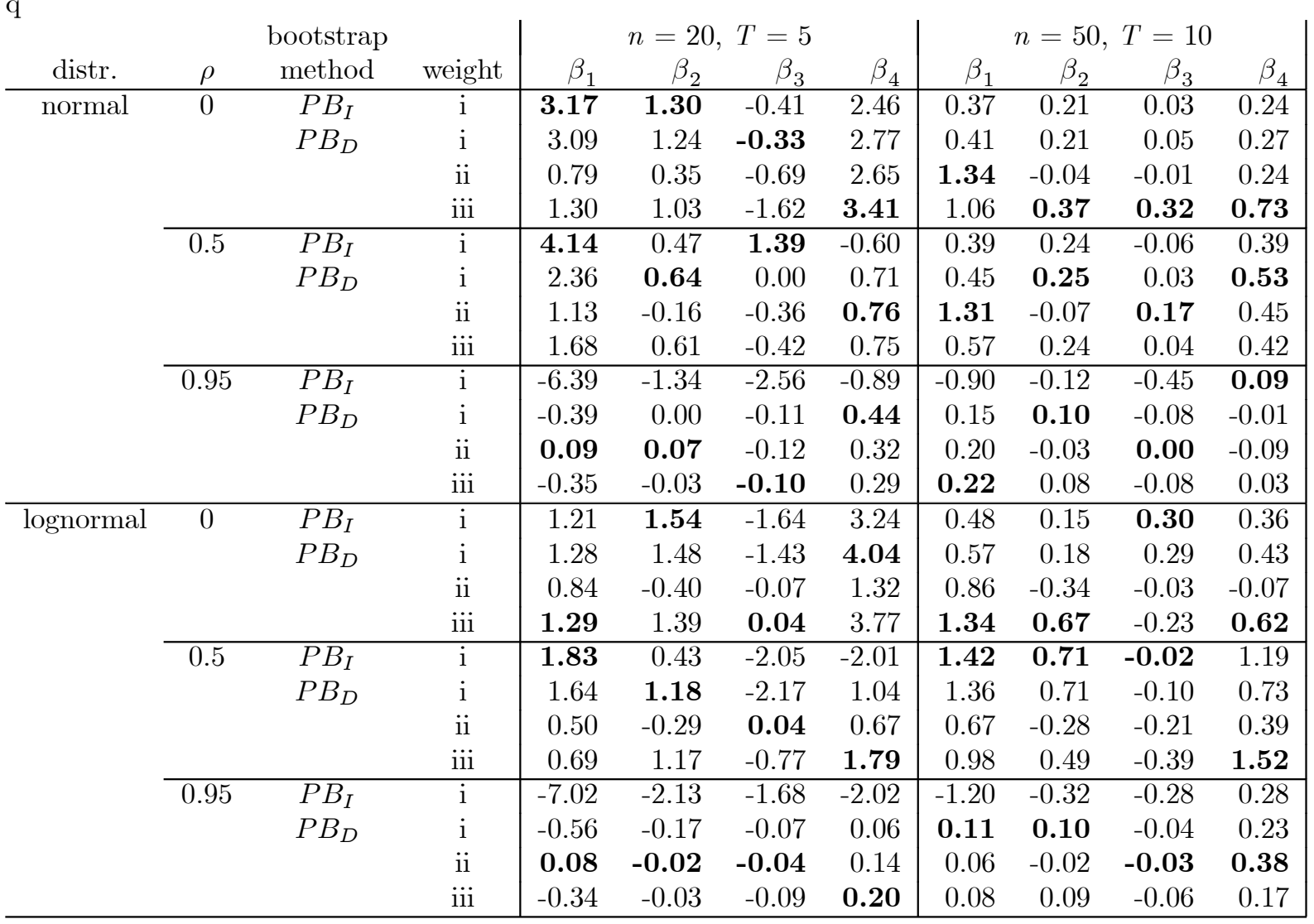

Table A.3: Confidence intervals, true coverage in percent for nominal 95 percent level, normal approximation without bias correction, mean values taken over all $\rho$

\begin{tabular}{|c|c|c|c|c|c|c|c|c|c|c|c|}
\hline \multirow{2}{*}{ distr. } & \multicolumn{3}{|c|}{ bootstrap } & \multicolumn{4}{|c|}{$n=20, T=5$} & \multicolumn{4}{|c|}{$n=50, T=10$} \\
\hline & $q$ & method & weight & $\beta_{1}$ & $\beta_{2}$ & $\beta_{3}$ & $\beta_{4}$ & $\beta_{1}$ & $\beta_{2}$ & $\beta_{3}$ & $\beta_{4}$ \\
\hline \multirow[t]{12}{*}{ normal } & 0.5 & $P B_{I}$ & $\mathrm{i}$ & 98.9 & 98.1 & 99.0 & 98.0 & 97.5 & 97.2 & 97.6 & 97.5 \\
\hline & & $P B_{D}$ & $\mathrm{i}$ & 97.2 & 96.9 & 97.8 & 95.5 & 96.5 & 96.3 & 97.3 & 96.7 \\
\hline & & & ii & 94.6 & 93.5 & 95.0 & 91.3 & 94.8 & 94.2 & 95.1 & 93.6 \\
\hline & & & iii & 97.4 & 97.8 & 98.2 & 96.6 & 97.8 & 97.9 & 98.5 & 98.2 \\
\hline & 0.75 & $P B_{I}$ & $\mathrm{i}$ & 98.6 & 98.1 & 98.9 & 97.7 & 97.7 & 97.5 & 98.0 & 97.7 \\
\hline & & $P B_{D}$ & $\mathrm{i}$ & 97.0 & 96.0 & 98.1 & 95.4 & 96.8 & 96.7 & 97.6 & 97.0 \\
\hline & & & ii & 95.6 & 92.5 & 96.2 & 93.1 & 96.7 & 94.6 & 96.7 & 95.8 \\
\hline & & & iii & 97.0 & 96.8 & 97.8 & 96.1 & 98.1 & 98.0 & 98.4 & 98.0 \\
\hline & 0.90 & $\overline{P B_{I}}$ & $\mathrm{i}$ & 97.7 & 97.0 & 98.5 & 98.0 & 97.8 & 96.8 & 98.0 & 98.0 \\
\hline & & $P B_{D}$ & $\mathrm{i}$ & 96.6 & 94.3 & 97.2 & 96.5 & 97.3 & 96.0 & 97.7 & 97.2 \\
\hline & & & ii & 95.8 & 91.4 & 96.0 & 94.3 & 97.3 & 93.9 & 97.3 & 96.1 \\
\hline & & & iii & 96.9 & 95.6 & 97.5 & 96.7 & 98.1 & 97.9 & 98.6 & 97.7 \\
\hline \multirow[t]{12}{*}{ lognormal } & 0.5 & $P B_{I}$ & $\mathrm{i}$ & 99.4 & 98.4 & 99.4 & 99.4 & 97.6 & 97.0 & 97.0 & 97.2 \\
\hline & & $P B_{D}$ & $\mathrm{i}$ & 98.4 & 96.6 & 98.0 & 97.2 & 96.5 & 95.5 & 96.7 & 96.5 \\
\hline & & & ii & 94.8 & 86.4 & 94.6 & 92.7 & 94.6 & 85.4 & 93.0 & 94.3 \\
\hline & & & iii & 98.3 & 97.0 & 98.0 & 97.2 & 97.8 & 97.3 & 97.8 & 98.0 \\
\hline & 0.75 & $P B_{I}$ & i & 98.7 & 98.4 & 98.7 & 98.3 & 98.0 & 97.2 & 97.6 & 97.9 \\
\hline & & $P B_{D}$ & $\mathrm{i}$ & 97.3 & 96.1 & 97.5 & 96.7 & 97.1 & 96.7 & 97.2 & 97.7 \\
\hline & & & ii & 96.3 & 80.5 & 95.8 & 93.7 & 97.1 & 80.5 & 95.5 & 95.4 \\
\hline & & & iii & 97.5 & 96.5 & 97.9 & 97.3 & 98.2 & 97.8 & 97.9 & 98.3 \\
\hline & 0.90 & $P B_{I}$ & $\mathrm{i}$ & 97.5 & 96.6 & 98.5 & 98.9 & 97.9 & 96.2 & 99.1 & 97.8 \\
\hline & & $P B_{D}$ & $\mathrm{i}$ & 96.4 & 94.2 & 97.3 & 97.7 & 97.6 & 95.5 & 98.7 & 97.1 \\
\hline & & & ii & 97.0 & 77.6 & 96.6 & 94.7 & 98.2 & 78.7 & 96.8 & 96.6 \\
\hline & & & iii & 97.1 & 95.1 & 97.7 & 98.0 & 98.0 & 96.9 & 98.9 & 97.0 \\
\hline
\end{tabular}


Table A.4: Confidence intervals, true coverage in percent for nominal 95 percent level, normal approximation with bias correction, mean values taken over all $\rho$

\begin{tabular}{|c|c|c|c|c|c|c|c|c|c|c|c|}
\hline & & $\begin{array}{l}\text { bootstral } \\
\text { method }\end{array}$ & weight & $\beta_{1}$ & $\begin{array}{c}n=2 \\
\beta_{2}\end{array}$ & $\begin{array}{c}T= \\
\beta_{3}\end{array}$ & $\beta_{4}$ & $\beta_{1}$ & $\begin{array}{c}=50 \\
\beta_{2}\end{array}$ & $\begin{array}{c}T= \\
\beta_{3}\end{array}$ & $\beta_{4}$ \\
\hline \multirow[t]{12}{*}{ normal } & \multirow[t]{4}{*}{0.5} & $P B_{I}$ & $\mathrm{i}$ & 97.0 & 96.8 & 98.0 & 93.5 & 96.4 & 95.9 & 96.4 & 96.3 \\
\hline & & $P B_{D}$ & i & 91.6 & 93.0 & 93.1 & 86.6 & 93.4 & 93.8 & 94.9 & 92.6 \\
\hline & & & ii & 86.6 & 86.7 & 88.2 & 78.2 & 91.0 & 90.7 & 92.0 & 88.1 \\
\hline & & & iii & 91.7 & 93.3 & 93.3 & 86.5 & 94.0 & 94.6 & 95.0 & 90.2 \\
\hline & \multirow[t]{4}{*}{0.75} & $P B_{I}$ & $\mathrm{i}$ & 96.5 & 97.1 & 97.4 & 93.0 & 96.2 & 95.9 & 96.9 & 96.2 \\
\hline & & $P B_{D}$ & $\mathrm{i}$ & 92.4 & 91.9 & 93.5 & 87.3 & 93.0 & 93.7 & 95.0 & 92.7 \\
\hline & & & ii & 87.4 & 86.0 & 89.8 & 79.8 & 92.8 & 91.0 & 93.3 & 90.3 \\
\hline & & & iii & 91.3 & 92.3 & 92.8 & 86.4 & 93.8 & 94.0 & 95.0 & 90.6 \\
\hline & \multirow[t]{4}{*}{0.90} & $P B_{I}$ & $\mathrm{i}$ & 94.4 & 95.2 & 96.4 & 92.4 & 95.9 & 95.4 & 97.2 & 95.2 \\
\hline & & $P B_{D}$ & $\mathrm{i}$ & 90.5 & 90.0 & 92.1 & 88.1 & 92.7 & 92.8 & 95.1 & 91.4 \\
\hline & & & ii & 87.3 & 83.5 & 88.0 & 80.8 & 91.9 & 89.4 & 93.1 & 89.1 \\
\hline & & & iii & 91.3 & 91.3 & 92.2 & 87.5 & 93.7 & 93.2 & 94.8 & 88.8 \\
\hline \multirow[t]{12}{*}{ lognormal } & \multirow[t]{4}{*}{0.5} & $P B_{I}$ & $\mathrm{i}$ & 98.4 & 97.2 & 98.7 & 96.9 & 96.6 & 95.3 & 95.7 & 96.1 \\
\hline & & $P B_{D}$ & i & 93.0 & 91.8 & 93.5 & 89.7 & 93.4 & 92.2 & 94.0 & 91.9 \\
\hline & & & ii & 86.1 & 79.1 & 85.5 & 80.1 & 89.2 & 80.7 & 88.6 & 88.3 \\
\hline & & & iii & 92.3 & 91.9 & 93.1 & 88.3 & 93.0 & 92.7 & 93.2 & 91.6 \\
\hline & \multirow[t]{4}{*}{0.75} & $P B_{I}$ & $\overline{\mathrm{i}}$ & 97.1 & 97.5 & 97.3 & 94.7 & 96.6 & 95.7 & 96.5 & 96.6 \\
\hline & & $P B_{D}$ & i & 92.9 & 93.0 & 93.0 & 88.8 & 93.5 & 93.5 & 95.0 & 93.0 \\
\hline & & & ii & 88.8 & 74.9 & 87.5 & 81.7 & 93.2 & 76.7 & 91.5 & 90.0 \\
\hline & & & iii & 92.5 & 92.9 & 92.7 & 89.1 & 94.1 & 94.2 & 94.8 & 90.9 \\
\hline & \multirow[t]{4}{*}{0.90} & $P B_{I}$ & $\mathrm{i}$ & 94.7 & 95.3 & 95.9 & 94.9 & 96.4 & 95.2 & 98.4 & 94.4 \\
\hline & & $P B_{D}$ & $\mathrm{i}$ & 91.5 & 90.9 & 91.3 & 91.6 & 93.1 & 93.2 & 96.0 & 89.4 \\
\hline & & & ii & 91.1 & 72.5 & 88.4 & 82.8 & 93.7 & 74.8 & 93.1 & 88.8 \\
\hline & & & iii & 91.6 & 91.6 & 91.0 & 91.2 & 93.7 & 93.6 & 95.6 & 86.1 \\
\hline
\end{tabular}

Table A.5: Bootstrap based confidence intervals, true coverage in percent for nominal 95 percent level, percentile method without bias correction, mean values taken over all $\rho$

\begin{tabular}{|c|c|c|c|c|c|c|c|c|c|c|c|}
\hline \multicolumn{4}{|c|}{ bootstrap } & \multicolumn{4}{|c|}{$n=20, T=5$} & \multicolumn{4}{|c|}{$n=50, T=10$} \\
\hline distr. & $q$ & method & weight & $\beta_{1}$ & $\beta_{2}$ & $\beta_{3}$ & $\beta_{4}$ & $\beta_{1}$ & $\beta_{2}$ & $\beta_{3}$ & $\beta_{4}$ \\
\hline \multirow[t]{12}{*}{ normal } & 0.5 & $P B_{I}$ & i & 99.6 & 99.0 & 99.3 & 99.3 & 98.0 & 97.3 & 97.1 & 97.8 \\
\hline & & $P B_{D}$ & $\mathrm{i}$ & 99.4 & 98.1 & 98.7 & 98.8 & 97.7 & 96.6 & 97.6 & 97.8 \\
\hline & & & ii & 98.2 & 95.3 & 97.2 & 97.4 & 95.9 & 94.3 & 95.2 & 95.7 \\
\hline & & & iii & 99.7 & 98.7 & 99.3 & 99.5 & 99.3 & 98.3 & 98.9 & 99.6 \\
\hline & 0.75 & $P B_{I}$ & $\mathrm{i}$ & 99.4 & 99.0 & 98.9 & 99.5 & 98.0 & 97.5 & 97.6 & 98.1 \\
\hline & & $P B_{D}$ & $\mathrm{i}$ & 98.9 & 97.9 & 98.5 & 99.2 & 97.5 & 97.3 & 97.8 & 98.2 \\
\hline & & & ii & 98.3 & 95.4 & 97.5 & 98.1 & 96.7 & 96.0 & 96.9 & 97.2 \\
\hline & & & iii & 99.6 & 98.6 & 98.9 & 99.5 & 99.4 & 99.0 & 99.2 & 99.5 \\
\hline & 0.90 & $P B_{I}$ & $\overline{\mathrm{i}}$ & 99.3 & 98.0 & 99.1 & 99.4 & 98.4 & 97.9 & $\overline{97.2}$ & 98.6 \\
\hline & & $P B_{D}$ & $\mathrm{i}$ & 98.3 & 95.5 & 98.7 & 99.0 & 98.1 & 97.4 & 97.6 & 98.5 \\
\hline & & & ii & 98.1 & 93.9 & 98.4 & 98.6 & 97.0 & 96.3 & 97.4 & 98.1 \\
\hline & & & iii & 99.0 & 97.3 & 99.1 & 99.6 & 99.4 & 99.1 & 99.1 & 99.6 \\
\hline \multirow[t]{12}{*}{ lognormal } & 0.5 & $P B_{I}$ & $\mathrm{i}$ & 99.5 & 99.3 & 99.3 & 99.4 & 98.0 & 97.7 & 97.2 & 97.8 \\
\hline & & $P B_{D}$ & $\mathrm{i}$ & 99.2 & 97.9 & 98.7 & 98.8 & 97.8 & 96.9 & 97.8 & 97.8 \\
\hline & & & ii & 97.6 & 90.7 & 96.7 & 96.9 & 96.4 & 90.1 & 94.9 & 96.2 \\
\hline & & & iii & 99.7 & 98.4 & 99.2 & 99.7 & 99.2 & 98.7 & 99.2 & 99.5 \\
\hline & 0.75 & $P B_{I}$ & $\mathrm{i}$ & 99.6 & 99.3 & 99.0 & 99.5 & 98.2 & 98.0 & 97.3 & 98.2 \\
\hline & & $P B_{D}$ & $\mathrm{i}$ & 99.1 & 97.4 & 98.5 & 99.4 & 98.2 & 97.4 & 97.5 & 98.3 \\
\hline & & & ii & 97.7 & 86.8 & 97.0 & 97.7 & 96.2 & 86.6 & 96.7 & 97.4 \\
\hline & & & iii & 99.5 & 98.2 & 98.9 & 99.8 & 99.5 & 99.0 & 99.0 & 99.6 \\
\hline & 0.90 & $P B_{I}$ & $\mathrm{i}$ & 99.7 & 98.4 & 98.8 & 99.6 & 98.3 & 97.4 & 96.7 & 98.5 \\
\hline & & $P B_{D}$ & $\mathrm{i}$ & 98.7 & 95.1 & 98.0 & 99.2 & 98.1 & 97.1 & 97.7 & 98.4 \\
\hline & & & ii & 96.5 & 82.2 & 98.1 & 98.5 & 94.7 & 85.4 & 97.4 & 98.2 \\
\hline & & & iii & 98.9 & 96.1 & 98.2 & 99.8 & 99.3 & 98.6 & 99.2 & 99.8 \\
\hline
\end{tabular}


Table A.6: Bootstrap based confidence intervals, true coverage in percent for nominal 95 percent level, percentile method with bias correction, mean values taken over all $\rho$

\begin{tabular}{|c|c|c|c|c|c|c|c|c|c|c|c|}
\hline \multirow{2}{*}{ distr. } & \multicolumn{3}{|c|}{ bootstrap } & \multicolumn{4}{|c|}{$n=20, T=5$} & \multicolumn{4}{|c|}{$n=50, T=10$} \\
\hline & $q$ & method & weight & $\beta_{1}$ & $\beta_{2}$ & $\beta_{3}$ & $\beta_{4}$ & $\beta_{1}$ & $\beta_{2}$ & $\beta_{3}$ & $\beta_{4}$ \\
\hline \multirow[t]{12}{*}{ normal } & 0.5 & $P B_{I}$ & $\mathrm{i}$ & 92.5 & 93.6 & 94.3 & 92.4 & 96.3 & 95.0 & 95.3 & 96.1 \\
\hline & & $P B_{D}$ & $\mathrm{i}$ & 83.9 & 87.4 & 87.2 & 82.0 & 93.1 & 92.2 & 94.0 & 92.1 \\
\hline & & & ii & 75.2 & 79.6 & 79.4 & 73.5 & 89.2 & 89.1 & 90.7 & 88.0 \\
\hline & & & iii & 81.7 & 86.5 & 85.9 & 80.2 & 89.7 & 90.5 & 91.0 & 89.3 \\
\hline & 0.75 & $P B_{I}$ & $\mathrm{i}$ & 90.1 & 92.7 & 93.2 & 91.7 & 95.8 & 95.7 & 96.0 & 96.0 \\
\hline & & $P B_{D}$ & $\mathrm{i}$ & 82.9 & 86.6 & 86.6 & 83.1 & 91.9 & 92.5 & 93.9 & 92.1 \\
\hline & & & ii & 76.0 & 80.8 & 81.1 & 74.9 & 90.3 & 90.4 & 91.7 & 90.1 \\
\hline & & & iii & 81.1 & 86.0 & 85.7 & 80.9 & 88.7 & 90.0 & 91.0 & 88.9 \\
\hline & 0.90 & $P B_{I}$ & $\mathrm{i}$ & 87.3 & 92.0 & 91.1 & 88.9 & 93.8 & 95.1 & 94.6 & 94.9 \\
\hline & & $P B_{D}$ & $\mathrm{i}$ & 81.4 & 85.3 & 84.5 & 82.0 & 89.3 & 91.6 & 91.9 & 90.3 \\
\hline & & & ii & 74.0 & 78.0 & 79.3 & 73.9 & 87.5 & 89.7 & 90.5 & 88.4 \\
\hline & & & iii & 80.5 & 86.3 & 84.2 & 80.0 & 86.3 & 89.1 & 89.7 & 86.3 \\
\hline \multirow[t]{12}{*}{ lognormal } & 0.5 & $P B_{I}$ & $\mathrm{i}$ & 96.2 & 95.8 & 97.0 & 96.2 & 96.5 & 95.8 & 95.7 & 96.0 \\
\hline & & $P B_{D}$ & $\mathrm{i}$ & 86.7 & 89.4 & 88.8 & 85.9 & 92.4 & 92.3 & 93.8 & 91.8 \\
\hline & & & ii & 77.3 & 77.7 & 80.1 & 75.8 & 87.8 & 84.8 & 89.6 & 88.5 \\
\hline & & & iii & 83.7 & 87.9 & 87.5 & 82.9 & 90.4 & 91.3 & 92.0 & 90.4 \\
\hline & 0.75 & $P B_{I}$ & $\mathrm{i}$ & 91.9 & 93.9 & 93.4 & 92.8 & 96.2 & 95.7 & 95.3 & 96.1 \\
\hline & & $P B_{D}$ & $\mathrm{i}$ & 84.2 & 88.2 & 85.6 & 84.1 & 92.1 & 92.9 & 93.4 & 91.9 \\
\hline & & & ii & 76.0 & 74.8 & 80.1 & 75.6 & 88.7 & 82.5 & 90.9 & 89.4 \\
\hline & & & iii & 81.1 & 87.5 & 84.6 & 81.2 & 89.3 & 90.8 & 91.1 & 88.1 \\
\hline & 0.90 & $P B_{I}$ & $\mathrm{i}$ & 86.9 & 90.7 & 90.0 & 87.7 & 92.2 & 92.5 & 92.7 & 93.8 \\
\hline & & $P B_{D}$ & $\mathrm{i}$ & 81.8 & 84.3 & 82.6 & 80.7 & 87.0 & 89.1 & 89.8 & 88.2 \\
\hline & & & ii & 77.2 & 72.6 & 80.3 & 75.3 & 81.5 & 79.7 & 89.9 & 86.3 \\
\hline & & & iii & 79.7 & 85.0 & 82.2 & 79.7 & 82.4 & 85.7 & 86.6 & 82.4 \\
\hline
\end{tabular}

Table A.7: Confidence intervals, true coverage in percent for nominal 95 percent level, normal approximation without bias correction, mean values taken over all quantiles q

\begin{tabular}{|c|c|c|c|c|c|c|c|c|c|c|c|}
\hline \multirow{2}{*}{ distr. } & \multicolumn{3}{|c|}{ bootstrap } & \multicolumn{4}{|c|}{$n=20, T=5$} & \multicolumn{4}{|c|}{$n=50, T=10$} \\
\hline & $\rho$ & method & weight & $\beta_{1}$ & $\beta_{2}$ & $\beta_{3}$ & $\beta_{4}$ & $\beta_{1}$ & $\beta_{2}$ & $\beta_{3}$ & $\beta_{4}$ \\
\hline \multirow[t]{12}{*}{ normal } & 0 & $P B_{I}$ & $\mathrm{i}$ & 96.4 & 95.6 & 97.4 & 95.6 & 96.4 & 96.4 & 97.7 & 97.0 \\
\hline & & $P B_{D}$ & $\mathrm{i}$ & 96.0 & 95.0 & 97.2 & 94.8 & 96.5 & 96.3 & 97.1 & 96.5 \\
\hline & & & ii & 94.5 & 90.8 & 95.6 & 91.5 & 95.9 & 94.8 & 96.6 & 94.4 \\
\hline & & & iii & 96.2 & 97.2 & 97.3 & 96.0 & 98.3 & 98.8 & 99.1 & 97.8 \\
\hline & 0.5 & $P B_{I}$ & $\mathrm{i}$ & 98.8 & 97.8 & 99.0 & 98.1 & 96.6 & 95.7 & 96.5 & 96.3 \\
\hline & & $P B_{D}$ & $\mathrm{i}$ & 97.0 & 96.3 & 97.8 & 95.4 & 96.7 & 96.2 & 97.8 & 96.5 \\
\hline & & & ii & 96.0 & 93.2 & 96.1 & 92.5 & 95.8 & 93.4 & 96.0 & 94.2 \\
\hline & & & iii & 97.5 & 96.7 & 98.0 & 96.3 & 98.1 & 98.2 & 98.7 & 98.0 \\
\hline & 0.95 & $P B_{I}$ & $\mathrm{i}$ & 100.0 & 99.8 & 100.0 & 100.0 & 99.9 & 99.4 & 99.4 & $\overline{99.9}$ \\
\hline & & $P B_{D}$ & $\mathrm{i}$ & 97.9 & 95.9 & 98.1 & 97.2 & 97.4 & 96.5 & 97.6 & 97.9 \\
\hline & & & ii & 95.5 & 93.4 & 95.4 & 94.7 & 97.1 & 94.5 & 96.5 & 96.9 \\
\hline & & & iii & 97.6 & 96.3 & 98.2 & 97.1 & 97.7 & 96.7 & 97.6 & 98.0 \\
\hline \multirow[t]{12}{*}{ lognormal } & 0 & $P B_{I}$ & $\mathrm{i}$ & 96.7 & 96.0 & 97.8 & 97.5 & 97.0 & 95.8 & 97.8 & 96.7 \\
\hline & & $P B_{D}$ & $\mathrm{i}$ & 96.6 & 95.4 & 97.5 & 96.8 & 96.8 & 95.7 & 97.3 & 96.3 \\
\hline & & & ii & 96.3 & 85.5 & 95.7 & 93.4 & 97.2 & 89.9 & 95.3 & 94.8 \\
\hline & & & iii & 97.4 & 96.9 & 97.7 & 97.4 & 98.5 & 98.3 & 98.5 & 97.6 \\
\hline & 0.5 & $P B_{I}$ & $\mathrm{i}$ & 98.9 & 98.0 & 98.8 & 99.1 & 96.6 & 95.4 & 96.0 & 96.2 \\
\hline & & $P B_{D}$ & $\mathrm{i}$ & 97.5 & 96.5 & 97.1 & 97.3 & 96.9 & 95.9 & 97.4 & 97.0 \\
\hline & & & ii & 97.7 & 79.3 & 95.2 & 94.3 & 97.0 & 81.7 & 93.2 & 94.9 \\
\hline & & & iii & 97.7 & 96.7 & 97.8 & 97.6 & 98.0 & 97.6 & 98.2 & 98.2 \\
\hline & 0.95 & $P B_{I}$ & $\mathrm{i}$ & 100.0 & 99.5 & 100.0 & 100.0 & 99.9 & 99.2 & 99.8 & 100.0 \\
\hline & & $P B_{D}$ & $\mathrm{i}$ & 97.9 & 95.0 & 98.1 & 97.4 & 97.5 & 96.0 & 98.0 & 97.9 \\
\hline & & & ii & 94.1 & 79.7 & 96.0 & 93.4 & 95.7 & 73.0 & 96.7 & 96.6 \\
\hline & & & iii & 97.8 & 95.0 & 98.1 & 97.5 & 97.5 & 96.1 & 97.9 & 97.5 \\
\hline
\end{tabular}


Table A.8: Confidence intervals, true coverage in percent for nominal 95 percent level, normal approximation with bias correction, mean values taken over all quantiles $q$

\begin{tabular}{|c|c|c|c|c|c|c|c|c|c|c|c|}
\hline \multirow{2}{*}{ distr. } & \multicolumn{3}{|c|}{ bootstrap } & \multicolumn{4}{|c|}{$n=20, T=5$} & \multicolumn{4}{|c|}{$n=50, T=10$} \\
\hline & $\rho$ & method & weight & $\beta_{1}$ & $\beta_{2}$ & $\beta_{3}$ & $\beta_{4}$ & $\beta_{1}$ & $\beta_{2}$ & $\beta_{3}$ & $\beta_{4}$ \\
\hline \multirow[t]{12}{*}{ normal } & 0 & $P B_{I}$ & $\mathrm{i}$ & 92.3 & 93.1 & 94.7 & 88.0 & 94.4 & 94.8 & 96.6 & 94.5 \\
\hline & & $P B_{D}$ & $\mathrm{i}$ & 91.7 & 91.8 & 94.4 & 87.1 & 94.1 & 94.5 & 96.2 & 94.1 \\
\hline & & & ii & 88.7 & 85.6 & 91.6 & 79.9 & 93.6 & 92.3 & 94.7 & 91.1 \\
\hline & & & iii & 91.4 & 93.6 & 94.1 & 86.0 & 95.3 & 95.4 & 96.2 & 89.8 \\
\hline & 0.5 & $P B_{I}$ & $\mathrm{i}$ & 95.7 & 96.4 & 97.2 & 91.7 & 94.8 & 93.8 & 95.3 & 93.9 \\
\hline & & $P B_{D}$ & $\mathrm{i}$ & 92.0 & 93.2 & 94.6 & 86.3 & 94.3 & 94.4 & 96.7 & 93.9 \\
\hline & & & ii & 89.0 & 86.8 & 91.3 & 79.8 & 93.4 & 90.9 & 94.1 & 90.6 \\
\hline & & & iii & 91.9 & 93.0 & 94.6 & 85.9 & 95.0 & 95.0 & 96.6 & 90.6 \\
\hline & 0.95 & $P B_{I}$ & i & 99.9 & 99.6 & 100.0 & 99.2 & 99.3 & 98.5 & 98.6 & 99.3 \\
\hline & & $P B_{D}$ & $\mathrm{i}$ & 90.8 & 89.8 & 89.6 & 88.6 & 90.8 & 91.5 & 92.1 & 88.7 \\
\hline & & & ii & 83.7 & 83.8 & 83.0 & 79.1 & 88.7 & 87.9 & 89.6 & 85.8 \\
\hline & & & iii & 91.0 & 90.2 & 89.6 & 88.5 & 91.2 & 91.4 & 92.0 & 89.2 \\
\hline \multirow[t]{12}{*}{ lognormal } & 0 & $P B_{I}$ & $\mathrm{i}$ & 93.3 & 93.8 & 94.8 & 91.4 & 94.9 & 94.3 & 96.5 & 94.3 \\
\hline & & $P B_{D}$ & $\mathrm{i}$ & 92.9 & 92.7 & 94.4 & 90.4 & 94.5 & 94.0 & 96.0 & 93.5 \\
\hline & & & ii & 91.6 & 80.8 & 91.3 & 83.5 & 95.0 & 86.7 & 93.1 & 91.1 \\
\hline & & & iii & 92.0 & 93.3 & 93.6 & 89.4 & 95.1 & 95.1 & 95.6 & 90.4 \\
\hline & 0.5 & $P B_{I}$ & $\mathrm{i}$ & $\overline{97.0}$ & 96.9 & 97.2 & 95.5 & 95.2 & 93.5 & 95.0 & 93.3 \\
\hline & & $P B_{D}$ & $\mathrm{i}$ & 92.3 & 92.4 & 94.3 & 89.5 & 95.3 & 94.0 & 96.4 & 93.5 \\
\hline & & & ii & 90.7 & 73.7 & 89.8 & 83.5 & 93.8 & 77.5 & 91.2 & 90.4 \\
\hline & & & iii & 91.7 & 92.6 & 93.6 & 88.3 & 95.0 & 94.7 & 95.6 & 91.6 \\
\hline & 0.95 & $P B_{I}$ & $\mathrm{i}$ & 99.9 & 99.2 & 99.9 & 99.6 & 99.5 & 98.3 & 99.1 & 99.4 \\
\hline & & $P B_{D}$ & i & 92.1 & 90.6 & 89.0 & 90.2 & 90.3 & 91.0 & 92.6 & 87.2 \\
\hline & & & ii & 83.7 & 72.1 & 80.3 & 77.6 & 87.4 & 67.9 & 88.9 & 85.6 \\
\hline & & & iii & 92.7 & 90.4 & 89.5 & 90.9 & 90.7 & 90.7 & 92.5 & 86.6 \\
\hline
\end{tabular}

Table A.9: Bootstrap based confidence intervals, true coverage in percent for nominal 95 percent level, percentile method without bias correction, mean values taken over all quantiles q

\begin{tabular}{|c|c|c|c|c|c|c|c|c|c|c|c|}
\hline \multirow{2}{*}{ distr. } & \multirow{2}{*}{\multicolumn{2}{|c|}{$\begin{array}{cc} & \text { bootstrap } \\
\rho & \text { method }\end{array}$}} & \multirow[b]{2}{*}{ weight } & \multicolumn{4}{|c|}{$n=20, T=5$} & \multicolumn{4}{|c|}{$n=50, T=10$} \\
\hline & & & & $\beta_{1}$ & $\beta_{2}$ & $\beta_{3}$ & $\beta_{4}$ & $\beta_{1}$ & $\beta_{2}$ & $\beta_{3}$ & $\beta_{4}$ \\
\hline & \multirow[t]{4}{*}{0} & $P B_{I}$ & i & 98.8 & 97.5 & 98.2 & 98.6 & 97.5 & 97.2 & 97.0 & 97.6 \\
\hline & & \multirow[t]{3}{*}{$P B_{D}$} & $\mathrm{i}$ & 98.3 & 96.5 & 97.8 & 98.3 & 97.1 & 96.6 & 97.1 & 97.5 \\
\hline & & & ii & 97.4 & 93.6 & 96.0 & 96.4 & 95.8 & 95.1 & 95.7 & 96.0 \\
\hline & & & iii & 99.7 & 98.8 & 98.8 & 99.4 & 99.7 & 99.4 & 99.5 & 99.6 \\
\hline & \multirow[t]{4}{*}{0.5} & $P B_{I}$ & $\mathrm{i}$ & 99.6 & 98.7 & 99.2 & 99.6 & 97.2 & 95.9 & 95.4 & 97.0 \\
\hline & & $P B_{D}$ & $\mathrm{i}$ & 99.3 & 97.3 & 98.3 & 98.9 & 97.3 & 96.6 & 97.1 & 97.4 \\
\hline & & & ii & 98.9 & 95.1 & 97.4 & 97.9 & 95.7 & 94.2 & 95.3 & 95.5 \\
\hline & & & iii & 99.7 & 98.1 & 98.7 & 99.4 & 99.4 & 98.8 & 98.8 & 99.5 \\
\hline & \multirow[t]{4}{*}{0.95} & $P B_{I}$ & $\bar{i}$ & 99.9 & 99.8 & 100.0 & 100.0 & 99.7 & 99.7 & 99.5 & $\overline{99.9}$ \\
\hline & & $P B_{D}$ & $\mathrm{i}$ & 99.0 & 97.6 & 99.7 & 99.8 & 99.0 & 98.1 & 98.8 & 99.6 \\
\hline & & & ii & 98.3 & 95.9 & 99.7 & 99.7 & 98.1 & 97.3 & 98.6 & 99.5 \\
\hline & & & iii & 99.0 & 97.8 & 99.8 & 99.8 & 99.0 & 98.1 & 98.9 & 99.6 \\
\hline \multirow[t]{12}{*}{ lognormal } & \multirow[t]{4}{*}{0} & $P B_{I}$ & $\mathrm{i}$ & 99.1 & 98.2 & 98.1 & 98.7 & 97.6 & 97.4 & 97.2 & 97.7 \\
\hline & & $P B_{D}$ & $\mathrm{i}$ & 98.6 & 97.1 & 97.6 & 98.3 & 97.5 & 96.5 & 97.0 & 97.2 \\
\hline & & & ii & 96.2 & 89.6 & 96.2 & 95.9 & 95.2 & 93.6 & 96.2 & 96.5 \\
\hline & & & iii & 99.6 & 98.8 & 98.3 & 99.6 & 99.7 & 99.4 & 99.4 & 99.6 \\
\hline & \multirow[t]{4}{*}{0.5} & $P B_{I}$ & i & 99.8 & 99.0 & 99.0 & 99.8 & 97.1 & 96.2 & 94.2 & 96.8 \\
\hline & & $P B_{D}$ & $\mathrm{i}$ & 99.7 & 97.5 & 98.1 & 99.3 & 97.2 & 96.9 & 96.7 & 97.5 \\
\hline & & & ii & 98.2 & 85.8 & 96.3 & 97.4 & 95.6 & 88.0 & 93.7 & 95.9 \\
\hline & & & iii & 99.8 & 97.9 & 98.4 & 99.8 & 99.2 & 98.9 & 98.6 & 99.4 \\
\hline & \multirow[t]{4}{*}{0.95} & $P B_{I}$ & $\mathrm{i}$ & 100.0 & 99.8 & 100.0 & 100.0 & 99.8 & 99.5 & 99.8 & 100.0 \\
\hline & & $P B_{D}$ & $\mathrm{i}$ & 98.7 & 95.8 & 99.6 & 99.8 & 99.3 & 98.0 & 99.3 & 99.7 \\
\hline & & & ii & 97.4 & 84.3 & 99.3 & 99.8 & 96.6 & 80.4 & 99.1 & 99.5 \\
\hline & & & iii & 98.8 & 96.0 & 99.7 & 99.9 & 99.2 & 98.1 & 99.4 & 99.8 \\
\hline
\end{tabular}


Table A.10: Bootstrap based confidence intervals, true coverage in percent for nominal 95 percent level, percentile method with bias correction, mean values taken over all quantiles $q$

\begin{tabular}{|c|c|c|c|c|c|c|c|c|c|c|c|}
\hline \multirow{2}{*}{ distr. } & \multicolumn{3}{|c|}{ bootstrap } & \multicolumn{4}{|c|}{$n=20, T=5$} & \multicolumn{4}{|c|}{$n=50, T=10$} \\
\hline & $\rho$ & method & weight & $\beta_{1}$ & $\beta_{2}$ & $\beta_{3}$ & $\beta_{4}$ & $\beta_{1}$ & $\beta_{2}$ & $\beta_{3}$ & $\beta_{4}$ \\
\hline \multirow[t]{12}{*}{ normal } & 0 & $P B_{I}$ & $\mathrm{i}$ & 84.0 & 88.0 & 88.2 & 84.5 & 94.1 & 94.5 & 94.7 & 94.5 \\
\hline & & $P B_{D}$ & $\mathrm{i}$ & 83.6 & 85.7 & 87.3 & 83.5 & 93.9 & 93.8 & 94.1 & 94.1 \\
\hline & & & ii & 76.0 & 77.8 & 81.2 & 74.3 & 91.9 & 91.6 & 92.5 & 91.2 \\
\hline & & & iii & 80.0 & 84.7 & 85.3 & 79.8 & 87.2 & 88.4 & 89.3 & 87.3 \\
\hline & 0.5 & $P B_{I}$ & $\mathrm{i}$ & 88.8 & 91.5 & 91.2 & 89.9 & 93.4 & 93.1 & 92.9 & 93.3 \\
\hline & & $P B_{D}$ & $\mathrm{i}$ & 81.8 & 86.3 & 86.0 & 82.7 & 92.9 & 92.8 & 94.5 & 93.0 \\
\hline & & & ii & 75.3 & 79.8 & 80.7 & 75.5 & 90.1 & 89.7 & 91.6 & 90.5 \\
\hline & & & iii & 80.9 & 86.5 & 86.0 & 79.9 & 89.8 & 91.0 & 90.9 & 89.2 \\
\hline & 0.95 & $P B_{I}$ & $\overline{\mathrm{i}}$ & 97.1 & 98.8 & 99.3 & 98.6 & 98.4 & 98.2 & 98.2 & 99.1 \\
\hline & & $P B_{D}$ & $\mathrm{i}$ & 82.8 & 87.4 & 85.0 & 80.9 & 87.5 & 89.6 & 91.2 & 87.4 \\
\hline & & & ii & 73.9 & 80.9 & 77.9 & 72.6 & 85.1 & 87.9 & 88.8 & 84.8 \\
\hline & & & iii & 82.5 & 87.6 & 84.5 & 81.4 & 87.7 & 90.3 & 91.5 & 88.0 \\
\hline \multirow[t]{12}{*}{ lognormal } & 0 & $P B_{I}$ & $\mathrm{i}$ & 85.2 & 88.5 & 88.6 & 85.7 & 93.6 & 93.6 & 94.3 & 94.1 \\
\hline & & $P B_{D}$ & $\mathrm{i}$ & 84.6 & 87.0 & 87.2 & 84.1 & 93.2 & 93.2 & 93.7 & 93.5 \\
\hline & & & ii & 77.8 & 78.4 & 82.3 & 77.2 & 89.0 & 88.9 & 92.1 & 90.5 \\
\hline & & & iii & 79.0 & 85.7 & 85.1 & 80.2 & 86.8 & 88.3 & 89.2 & 86.7 \\
\hline & 0.5 & $P B_{I}$ & $\overline{\mathrm{i}}$ & 91.2 & 93.2 & 92.5 & 91.5 & 93.0 & 92.4 & 91.3 & 92.5 \\
\hline & & $P B_{D}$ & $\mathrm{i}$ & 83.4 & 87.4 & 87.0 & 83.6 & 92.5 & 92.4 & 93.4 & 92.7 \\
\hline & & & ii & 77.5 & 73.8 & 83.3 & 77.5 & 88.1 & 83.3 & 90.4 & 89.5 \\
\hline & & & iii & 80.7 & 86.2 & 85.8 & 80.6 & 88.9 & 90.8 & 90.5 & 88.8 \\
\hline & 0.95 & $P B_{I}$ & $\mathrm{i}$ & 98.6 & 98.7 & 99.3 & 99.4 & 98.3 & 98.0 & 98.1 & 99.3 \\
\hline & & $P B_{D}$ & $\mathrm{i}$ & 84.6 & 87.5 & 82.8 & 83.0 & 85.9 & 88.7 & 89.8 & 85.7 \\
\hline & & & ii & 75.2 & 73.0 & 74.9 & 72.1 & 80.9 & 74.8 & 87.9 & 84.2 \\
\hline & & & iii & 84.8 & 88.5 & 83.4 & 82.9 & 86.5 & 88.7 & 90.0 & 85.4 \\
\hline
\end{tabular}

Table A.11: Median length of bootstrap based confidence intervals for nominal 95 percent level, normal approximation method, mean values taken over all $\rho$

\begin{tabular}{|c|c|c|c|c|c|c|c|c|c|c|c|}
\hline \multirow[b]{2}{*}{ distr. } & \multicolumn{3}{|c|}{ bootstrap } & \multicolumn{4}{|c|}{$n=20, T=5$} & \multicolumn{4}{|c|}{$n=50, T=10$} \\
\hline & $q$ & method & weight & $\beta_{1}$ & $\beta_{2}$ & $\beta_{3}$ & $\beta_{4}$ & $\beta_{1}$ & $\beta_{2}$ & $\beta_{3}$ & $\beta_{4}$ \\
\hline \multirow[t]{12}{*}{ normal } & 0.5 & $P B_{I}$ & i & 13.08 & 5.10 & 4.25 & 0.59 & 2.55 & 1.79 & 0.91 & 0.28 \\
\hline & & $P B_{D}$ & $\mathrm{i}$ & 7.61 & 3.33 & 2.67 & 0.43 & 2.18 & 1.59 & 0.85 & 0.24 \\
\hline & & & ii & 5.98 & 2.82 & 2.13 & 0.39 & 2.06 & 1.51 & 0.79 & 0.23 \\
\hline & & & iii & 6.86 & 3.44 & 2.55 & 0.43 & 2.87 & 2.02 & 1.08 & 0.28 \\
\hline & 0.75 & $P B_{I}$ & $\mathrm{i}$ & 13.47 & 5.59 & 4.43 & 0.61 & 2.85 & 1.98 & 1.00 & 0.30 \\
\hline & & $P B_{D}$ & $\mathrm{i}$ & 7.80 & 3.65 & 2.81 & 0.45 & 2.45 & 1.79 & 0.94 & 0.27 \\
\hline & & & ii & 6.41 & 3.04 & 2.29 & 0.41 & 2.28 & 1.67 & 0.87 & 0.25 \\
\hline & & & iii & 7.03 & 3.82 & 2.67 & 0.44 & 3.22 & 2.32 & 1.21 & 0.31 \\
\hline & 0.90 & $P B_{I}$ & $\mathrm{i}$ & 12.43 & 6.29 & 4.38 & 0.64 & 4.09 & 2.67 & 1.41 & 0.37 \\
\hline & & $P B_{D}$ & $\mathrm{i}$ & 8.09 & 4.36 & 3.03 & 0.47 & 3.43 & 2.42 & 1.31 & 0.33 \\
\hline & & & ii & 6.66 & 3.52 & 2.50 & 0.44 & 3.04 & 2.12 & 1.14 & 0.31 \\
\hline & & & iii & 7.00 & 4.64 & 2.85 & 0.47 & 4.29 & 3.15 & 1.66 & 0.38 \\
\hline \multirow[t]{12}{*}{ lognormal } & 0.5 & $P B_{I}$ & $\mathrm{i}$ & 7.40 & 2.96 & 2.47 & 0.41 & 1.54 & 1.11 & 0.56 & 0.18 \\
\hline & & $P B_{D}$ & $\mathrm{i}$ & 3.32 & 1.72 & 1.25 & 0.26 & 1.25 & 0.94 & 0.51 & 0.15 \\
\hline & & & ii & 2.31 & 1.37 & 0.89 & 0.21 & 1.12 & 0.86 & 0.44 & 0.13 \\
\hline & & & iii & 3.14 & 1.73 & 1.22 & 0.26 & 1.43 & 1.08 & 0.59 & 0.17 \\
\hline & 0.75 & $P B_{I}$ & $\mathrm{i}$ & 13.43 & 6.59 & 4.56 & 0.64 & 2.88 & 2.01 & 1.02 & 0.30 \\
\hline & & $P B_{D}$ & $\mathrm{i}$ & 7.44 & 3.87 & 2.79 & 0.43 & 2.41 & 1.79 & 0.95 & 0.26 \\
\hline & & & ii & 3.44 & 1.88 & 1.32 & 0.28 & 1.67 & 1.26 & 0.66 & 0.20 \\
\hline & & & iii & 6.27 & 3.70 & 2.49 & 0.40 & 3.01 & 2.23 & 1.18 & 0.30 \\
\hline & 0.90 & $P B_{I}$ & $\mathrm{i}$ & 16.91 & 14.40 & 6.81 & 1.31 & 9.39 & 6.30 & 3.38 & 0.57 \\
\hline & & $P B_{D}$ & $\mathrm{i}$ & 11.50 & 10.28 & 5.03 & 0.97 & 7.99 & 5.66 & 3.10 & 0.52 \\
\hline & & & ii & 5.23 & 3.09 & 2.02 & 0.38 & 3.12 & 2.28 & 1.20 & 0.32 \\
\hline & & & iii & 9.15 & 9.03 & 4.31 & 0.78 & 8.29 & 6.62 & 3.30 & 0.57 \\
\hline
\end{tabular}


Table A.12: Median length of bootstrap based confidence intervals for nominal 95 percent level, percentile method without bias correction, mean values taken over all $\rho$

\begin{tabular}{|c|c|c|c|c|c|c|c|c|c|c|c|}
\hline \multirow[b]{2}{*}{ distr. } & \multicolumn{3}{|c|}{ bootstrap } & \multicolumn{4}{|c|}{$n=20, T=5$} & \multicolumn{4}{|c|}{$n=50, T=10$} \\
\hline & $q$ & method & weight & $\beta_{1}$ & $\beta_{2}$ & $\beta_{3}$ & $\beta_{4}$ & $\beta_{1}$ & $\beta_{2}$ & $\beta_{3}$ & $\beta_{4}$ \\
\hline \multirow[t]{12}{*}{ normal } & 0.5 & $P D_{I}$ & $\mathrm{i}$ & 10.74 & 4.32 & 3.76 & 0.62 & 2.51 & 1.77 & 0.91 & 0.28 \\
\hline & & $P D_{D}$ & $\mathrm{i}$ & 6.44 & 3.02 & 2.40 & 0.44 & 2.16 & 1.57 & 0.85 & 0.25 \\
\hline & & & ii & 5.01 & 2.60 & 1.89 & 0.40 & 2.04 & 1.50 & 0.79 & 0.23 \\
\hline & & & iii & 5.69 & 3.02 & 2.23 & 0.44 & 2.57 & 1.86 & 1.01 & 0.29 \\
\hline & 0.75 & $P D_{I}$ & $\mathrm{i}$ & 10.91 & 4.53 & 3.95 & 0.64 & 2.77 & 1.94 & 1.00 & 0.31 \\
\hline & & $P D_{D}$ & $\mathrm{i}$ & 6.61 & 3.23 & 2.55 & 0.46 & 2.40 & 1.77 & 0.93 & 0.27 \\
\hline & & & ii & 5.27 & 2.74 & 2.04 & 0.42 & 2.24 & 1.66 & 0.86 & 0.25 \\
\hline & & & iii & 5.78 & 3.25 & 2.35 & 0.46 & 2.81 & 2.10 & 1.11 & 0.31 \\
\hline & 0.90 & $P D_{I}$ & $\mathrm{i}$ & 10.28 & 5.15 & 4.01 & 0.67 & 3.72 & 2.52 & 1.35 & 0.38 \\
\hline & & $P D_{D}$ & $\mathrm{i}$ & 6.60 & 3.80 & 2.77 & 0.49 & 3.17 & 2.32 & 1.25 & 0.34 \\
\hline & & & ii & 5.42 & 3.06 & 2.22 & 0.45 & 2.89 & 2.05 & 1.11 & 0.31 \\
\hline & & & iii & 5.79 & 3.84 & 2.64 & 0.48 & 3.64 & 2.71 & 1.46 & 0.38 \\
\hline \multirow[t]{12}{*}{ lognormal } & 0.5 & $P D_{I}$ & $\overline{\mathrm{i}}$ & 6.11 & 2.68 & 2.15 & 0.43 & 1.54 & 1.11 & 0.56 & 0.18 \\
\hline & & $P D_{D}$ & $\mathrm{i}$ & 3.04 & 1.65 & 1.17 & 0.27 & 1.25 & 0.95 & 0.51 & 0.15 \\
\hline & & & ii & 2.26 & 1.32 & 0.86 & 0.22 & 1.13 & 0.85 & 0.44 & 0.14 \\
\hline & & & iii & 2.89 & 1.65 & 1.15 & 0.27 & 1.43 & 1.07 & 0.59 & 0.17 \\
\hline & 0.75 & $P D_{I}$ & $\overline{\mathrm{i}}$ & 11.02 & 4.72 & 4.07 & 0.65 & 2.78 & 1.96 & $\overline{1.00}$ & 0.31 \\
\hline & & $P D_{D}$ & $\mathrm{i}$ & 6.18 & 3.17 & 2.49 & 0.43 & 2.35 & 1.73 & 0.94 & 0.27 \\
\hline & & & ii & 3.08 & 1.74 & 1.20 & 0.28 & 1.68 & 1.24 & 0.66 & 0.20 \\
\hline & & & iii & 5.16 & 3.08 & 2.21 & 0.41 & 2.71 & 2.01 & 1.09 & 0.30 \\
\hline & 0.90 & $P D_{I}$ & $\mathrm{i}$ & 14.47 & 11.47 & 6.88 & 1.06 & 7.21 & 4.74 & 2.70 & 0.58 \\
\hline & & $P D_{D}$ & $\mathrm{i}$ & 9.76 & 8.41 & 5.03 & 0.76 & 6.23 & 4.33 & 2.55 & 0.52 \\
\hline & & & ii & 4.31 & 2.61 & 1.81 & 0.39 & 2.92 & 2.11 & 1.15 & 0.32 \\
\hline & & & iii & 7.77 & 7.35 & 4.23 & 0.66 & 6.43 & 4.83 & 2.73 & 0.56 \\
\hline
\end{tabular}

Table A.13: Median length of bootstrap based confidence intervals for nominal 95 percent level, normal approximation method, mean values taken over all quantiles $\mathrm{q}$

\begin{tabular}{|c|c|c|c|c|c|c|c|c|c|c|c|}
\hline \multirow{2}{*}{ distr. } & \multicolumn{3}{|c|}{ bootstrap } & \multicolumn{4}{|c|}{$n=20, T=5$} & \multicolumn{4}{|c|}{$n=50, T=10$} \\
\hline & $\rho$ & method & weight & $\beta_{1}$ & $\beta_{2}$ & $\beta_{3}$ & $\beta_{4}$ & $\beta_{1}$ & $\beta_{2}$ & $\beta_{3}$ & $\beta_{4}$ \\
\hline \multirow[t]{12}{*}{ normal } & 0 & $P D_{I}$ & $\mathrm{i}$ & 13.36 & 5.88 & 4.47 & 0.65 & 3.29 & 2.19 & 1.14 & 0.32 \\
\hline & & $P D_{D}$ & $\mathrm{i}$ & 12.39 & 5.37 & 4.23 & 0.63 & 3.15 & 2.15 & 1.12 & 0.32 \\
\hline & & & ii & 9.41 & 4.13 & 3.27 & 0.55 & 2.89 & 1.97 & 1.03 & 0.30 \\
\hline & & & iii & 11.06 & 5.90 & 4.07 & 0.64 & 4.81 & 3.32 & 1.71 & 0.40 \\
\hline & 0.5 & $P D_{I}$ & $\mathrm{i}$ & 13.40 & 5.82 & 4.52 & 0.63 & 3.16 & 2.17 & 1.11 & 0.32 \\
\hline & & $P D_{D}$ & $\mathrm{i}$ & 8.66 & 4.15 & 3.23 & 0.51 & 3.14 & 2.23 & 1.21 & 0.33 \\
\hline & & & ii & 7.13 & 3.41 & 2.61 & 0.46 & 2.79 & 1.98 & 1.07 & 0.30 \\
\hline & & & iii & 7.41 & 4.18 & 2.94 & 0.49 & 3.81 & 2.76 & 1.48 & 0.37 \\
\hline & 0.95 & $P D_{I}$ & $\overline{\mathrm{i}}$ & 12.21 & 5.28 & 4.06 & 0.56 & 3.04 & 2.08 & 1.08 & 0.30 \\
\hline & & $P D_{D}$ & $\mathrm{i}$ & 2.44 & 1.82 & 1.06 & 0.21 & 1.77 & 1.42 & 0.77 & 0.20 \\
\hline & & & ii & 2.50 & 1.85 & 1.03 & 0.22 & 1.70 & 1.36 & 0.70 & 0.19 \\
\hline & & & iii & 2.42 & 1.82 & 1.06 & 0.21 & 1.77 & 1.42 & 0.77 & 0.20 \\
\hline \multirow[t]{12}{*}{ lognormal } & 0 & $P D_{I}$ & $\overline{\mathrm{i}}$ & 12.03 & 8.45 & 4.54 & 0.90 & 5.01 & 3.39 & 1.80 & 0.35 \\
\hline & & $P D_{D}$ & $\mathrm{i}$ & 11.36 & 8.00 & 4.36 & 0.90 & 4.78 & 3.30 & 1.75 & 0.34 \\
\hline & & & ii & 4.96 & 2.55 & 1.82 & 0.37 & 2.32 & 1.66 & 0.85 & 0.24 \\
\hline & & & iii & 9.32 & 7.15 & 3.79 & 0.73 & 5.73 & 4.50 & 2.13 & 0.42 \\
\hline & 0.5 & $P D_{I}$ & $\overline{\mathrm{i}}$ & 12.89 & 8.48 & 4.88 & 0.82 & 4.70 & 3.28 & 1.73 & 0.36 \\
\hline & & $P D_{D}$ & $\mathrm{i}$ & 8.63 & 5.99 & 3.73 & 0.59 & 4.87 & 3.49 & 1.92 & 0.38 \\
\hline & & & ii & 4.07 & 2.19 & 1.65 & 0.34 & 2.08 & 1.55 & 0.83 & 0.24 \\
\hline & & & iii & 6.98 & 5.43 & 3.25 & 0.53 & 5.00 & 3.84 & 2.04 & 0.41 \\
\hline & 0.95 & $P D_{I}$ & $\mathrm{i}$ & 12.82 & 7.03 & 4.42 & 0.63 & 4.11 & 2.76 & 1.43 & 0.34 \\
\hline & & $P D_{D}$ & $\mathrm{i}$ & 2.27 & 1.89 & 0.98 & 0.18 & 2.01 & 1.60 & 0.90 & 0.21 \\
\hline & & & ii & 1.95 & 1.58 & 0.76 & 0.16 & 1.50 & 1.18 & 0.63 & 0.16 \\
\hline & & & iii & 2.26 & 1.88 & 0.98 & 0.18 & 1.99 & 1.60 & 0.89 & 0.21 \\
\hline
\end{tabular}


Table A.14: Median length of bootstrap based confidence intervals for nominal 95 percent level, percentile method without bias correction, mean values taken over all quantiles q

\begin{tabular}{|c|c|c|c|c|c|c|c|c|c|c|c|}
\hline \multirow{2}{*}{ distr. } & \multicolumn{3}{|c|}{ bootstrap } & \multicolumn{4}{|c|}{$n=20, T=5$} & \multicolumn{4}{|c|}{$n=50, T=10$} \\
\hline & $\rho$ & method & weight & $\beta_{1}$ & $\beta_{2}$ & $\beta_{3}$ & $\beta_{4}$ & $\beta_{1}$ & $\beta_{2}$ & $\beta_{3}$ & $\beta_{4}$ \\
\hline \multirow[t]{12}{*}{ normal } & \multirow[t]{4}{*}{0} & \multirow{4}{*}{$\begin{array}{l}P D_{I} \\
P D_{D}\end{array}$} & $\mathrm{i}$ & 10.98 & 4.84 & 4.04 & 0.66 & 3.08 & 2.12 & 1.11 & 0.33 \\
\hline & & & i & 10.28 & 4.57 & 3.85 & 0.64 & 2.99 & 2.08 & 1.09 & 0.32 \\
\hline & & & ii & 7.52 & 3.53 & 2.84 & 0.56 & 2.77 & 1.92 & 1.01 & 0.30 \\
\hline & & & iii & 8.85 & 4.73 & 3.55 & 0.65 & 3.85 & 2.77 & 1.44 & 0.41 \\
\hline & \multirow[t]{4}{*}{0.5} & $P D_{I}$ & $\mathrm{i}$ & 11.04 & 4.79 & 4.08 & 0.65 & 3.00 & 2.09 & 1.09 & 0.33 \\
\hline & & \multirow{3}{*}{$P D_{D}$} & $\mathrm{i}$ & 6.91 & 3.69 & 2.81 & 0.52 & 2.96 & 2.17 & 1.17 & 0.33 \\
\hline & & & ii & 5.71 & 3.08 & 2.30 & 0.47 & 2.69 & 1.94 & 1.05 & 0.30 \\
\hline & & & iii & 5.95 & 3.59 & 2.62 & 0.51 & 3.38 & 2.49 & 1.37 & 0.37 \\
\hline & \multirow[t]{4}{*}{0.95} & $P D_{I}$ & $\mathrm{i}$ & 9.90 & 4.37 & 3.60 & 0.62 & 2.90 & 2.02 & 1.05 & 0.31 \\
\hline & & \multirow{3}{*}{$P D_{D}$} & $\mathrm{i}$ & 2.46 & 1.79 & 1.05 & 0.23 & 1.78 & 1.41 & 0.77 & 0.20 \\
\hline & & & ii & 2.47 & 1.80 & 1.01 & 0.23 & 1.72 & 1.35 & 0.70 & 0.19 \\
\hline & & & iii & 2.47 & 1.79 & 1.06 & 0.23 & 1.79 & 1.41 & 0.77 & 0.20 \\
\hline \multirow[t]{12}{*}{ lognormal } & \multirow[t]{4}{*}{0} & $P D_{I}$ & $\mathrm{i}$ & 10.03 & 6.99 & 4.42 & 0.72 & 4.08 & 2.67 & 1.51 & 0.35 \\
\hline & & \multirow{3}{*}{$P D_{D}$} & $\mathrm{i}$ & 9.53 & 6.39 & 4.16 & 0.69 & 3.90 & 2.64 & 1.47 & 0.34 \\
\hline & & & ii & 4.12 & 2.18 & 1.62 & 0.38 & 2.20 & 1.56 & 0.81 & 0.24 \\
\hline & & & iii & 7.65 & 5.84 & 3.57 & 0.63 & 4.49 & 3.30 & 1.75 & 0.41 \\
\hline & \multirow[t]{4}{*}{0.5} & $P D_{I}$ & $\mathrm{i}$ & 10.92 & 6.53 & 4.64 & 0.74 & 3.90 & 2.67 & 1.46 & 0.36 \\
\hline & & \multirow{3}{*}{$P D_{D}$} & i & 7.20 & 5.02 & 3.56 & 0.57 & 3.94 & 2.80 & 1.65 & 0.38 \\
\hline & & & ii & 3.59 & 1.98 & 1.50 & 0.34 & 2.01 & 1.48 & 0.81 & 0.25 \\
\hline & & & iii & 5.93 & 4.42 & 3.05 & 0.52 & 4.10 & 3.05 & 1.76 & 0.41 \\
\hline & \multirow[t]{4}{*}{0.95} & $P D_{I}$ & $\mathrm{i}$ & 10.65 & 5.36 & 4.04 & 0.68 & 3.55 & 2.47 & 1.30 & 0.35 \\
\hline & & \multirow{3}{*}{$P D_{D}$} & i & 2.25 & 1.82 & 0.98 & 0.19 & 2.00 & 1.56 & 0.89 & 0.22 \\
\hline & & & ii & 1.93 & 1.51 & 0.75 & 0.16 & 1.51 & 1.16 & 0.63 & 0.17 \\
\hline & & & iii & 2.24 & 1.82 & 0.98 & 0.19 & 1.99 & 1.56 & 0.89 & 0.21 \\
\hline
\end{tabular}

